\title{
Generation of Tubular and Membranous Shape Textures with Curvature Functionals
}

\author{
Anna Song ${ }^{1,2}$ (D) \\ Received: 6 March 2021 / Accepted: 11 July 2021 / Published online: 19 August 2021 \\ (c) The Author(s) 2021
}

\begin{abstract}
Tubular and membranous shapes display a wide range of morphologies that are difficult to analyze within a common framework. By generalizing the classical Helfrich energy of biomembranes, we model them as solutions to a curvature optimization problem in which the principal curvatures may play asymmetric roles. We then give a novel phase-field formulation to approximate this geometric problem, and study its Gamma-limsup convergence. This results in an efficient GPU algorithm that we validate on well-known minimizers of the Willmore energy; the software for the implementation of our algorithm is freely available online. Exploring the space of parameters reveals that this comprehensive framework leads to a wide continuum of shape textures. This first step towards a unifying theory will have several implications, in biology for quantifying tubular shapes or designing bio-mimetic scaffolds, but also in computer graphics, materials science, or architecture.
\end{abstract}

Keywords Tubular shapes · Curvature functionals · Phase-fields · Biomembranes · Gamma-limsup

\section{Introduction}

Tubular and membranous shape textures are widely present in biology. They display a large variety of morphologies in terms of geometry and topology, which are important to analyze since they reflect the state of a biological system. For instance, the bone marrow capillaries are highly branching and merging vessels [88,101], whose organization is subject to drastic remodeling in acute myeloid leukaemia $[30,82]$. In cells, the endoplasmic reticulum, where proteins are synthesized, consists of an interconnected network [96] that undergoes sheets-to-tubules topological transformations [87]. Furthermore, trabecular bone is a combination of rods and platelets $[76,93]$ that are optimally restructured under mechanical stress $[1,90,92]$ or pathological conditions [40,81,103].

However, due to their disparity and complexity, tubular and membranous structures are difficult to describe within a unifying framework that captures both their rich morpho-

Anna Song

a.song19@imperial.ac.uk

1 Department of Mathematics, Imperial College London, London, UK

2 Haematopoietic Stem Cell Laboratory, The Francis Crick Institute, London, UK logical diversity as well as their continuous variations. We approach this question by building a generation model that creates shape textures from noise, similarly to texture synthesis in images $[46,62,85]$. We model tubules and membranes as optimizers under constant volume of a curvature functional

$\mathbf{F}(\mathscr{S})=\int_{\mathscr{S}} p\left(\kappa_{1}, \kappa_{2}\right) \mathrm{d} A$,

where $p$ is a second-degree polynomial of the principal curvatures $\kappa_{1}$ and $\kappa_{2}$ of the surface $\mathscr{S}$. As our main contribution, we provide a novel phase-field formulation $\mathscr{F}_{\epsilon}$ to approximate the original geometric problem $\mathbf{F}$, and show that the $\Gamma$-limsup holds, a notion coming from the $\Gamma$-convergence framework $[3,12,24]$. The optimization problem then translates into the mass-preserving $H^{-1}$ gradient flow $[22,35]$

$\dot{u}=\Delta \frac{\partial \mathscr{F}_{\epsilon}}{\partial u}(u)$.

Combining the stochastic optimizer Adam [55,66] to the automatic differentiation provided by PyTorch [83] results in an efficient and flexible GPU implementation, curvatubes. It successfully leads to a wide continuum of shape textures (see Fig. 1), which constitutes a first step towards a unifying theory. 


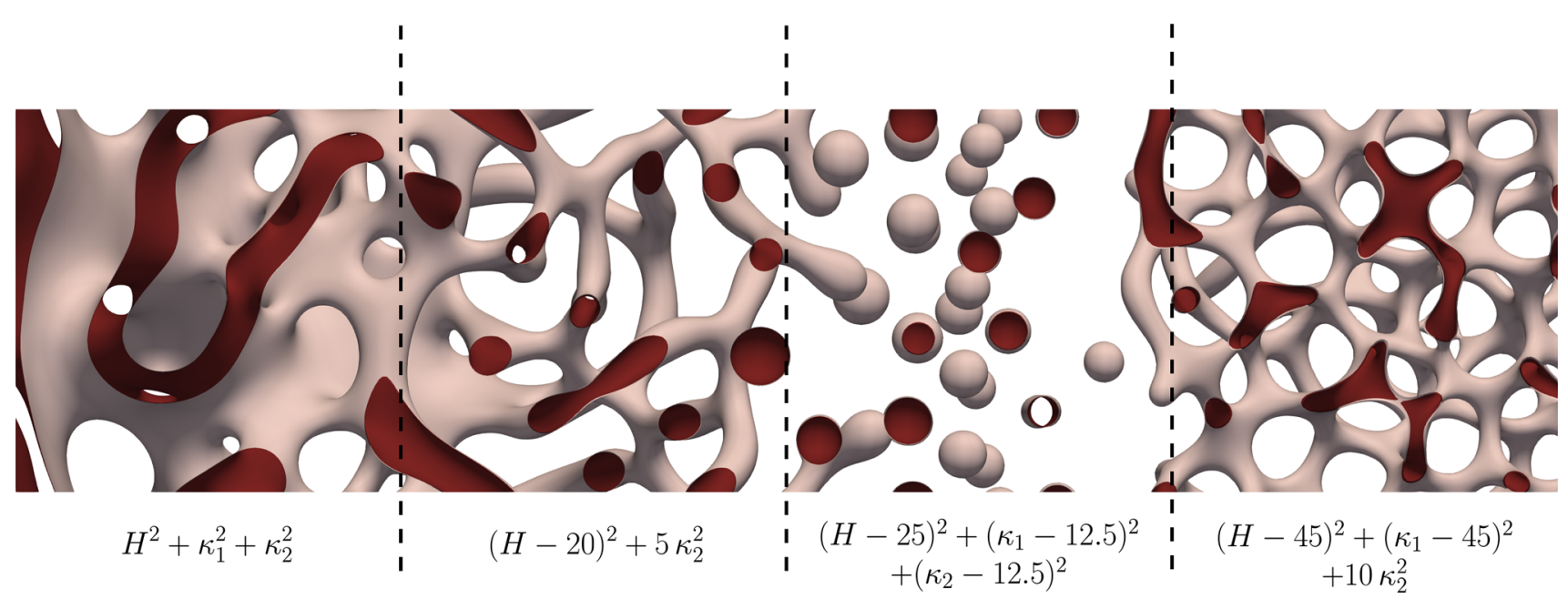

Fig. 1 A continuum of shape textures generated by curvatubes, after optimizing a curvature-based polynomial energy of the surface $\int_{\mathscr{S}} p\left(\kappa_{1}, \kappa_{2}\right) \mathrm{d} A$, with a volume constraint. The polynomial coefficients vary linearly in space, by interpolating four values that define different shape textures, at the vertical median of the four squares. In the formulas, $\kappa_{1}$ and $\kappa_{2}$ are the principal curvatures of the surface, while $H=\kappa_{1}+\kappa_{2}$ and $K=\kappa_{1} \kappa_{2}$ are the mean and the Gaussian curvatures. More details are given at the end of Sect. 5.2
Related work. The variational formulation (1) generalizes two classical functionals, the Willmore energy studied in differential geometry $[6,14,51,67,100,104,110]$, as well as the Helfrich energy used to model biomembranes [17,18,25, $28,44,97]$. In contrast to these models, the polynomial $p$ is here not required to be symmetric in the principal curvatures, which allows the generation of tubules. Other generalizations have been proposed in [43,106] and [19,26].

Curvature functionals are often used as image prior models in imaging, due to their ability to interpolate. Mumford [75] considered Euler's elastica functional as a prior curve model in computer vision, and this was subsequently applied to digital inpainting $[10,68,99]$. Similar ideas were then used for $3 \mathrm{D}$ volume reconstruction from $2 \mathrm{D}$ slices $[13,53,64]$. Our work is also related to Poisson reconstruction methods [48, 49] that implicitly reconstruct a surface from noisy oriented points.

However, as in [27-29], we use the diffuse setting to model shapes. By construction, our phase-field formulation $\mathscr{F}_{\epsilon}$ extends the standard approximation of the Willmore energy (see [14] and references therein) as well as the one proposed by Bellettini \& Mugnai in [8] for the Helfrich energy. In terms of $\Gamma$-convergence, we also provide an extension of the $\Gamma$-limsup result in [8], while keeping the $\Gamma$-liminf as an open question (except in the cases previously covered) that we do not aim to solve here.

Finally, our attempt in building a unifying framework is connected to the Functionalized Cahn-Hilliard (FCH) model proposed in $[20,21,23,41,56,57]$. The FCH energy describes how amphiphilic molecules self-assemble into complex network morphologies that feature spheres, tubules, sheets, and mixtures of them. Loosely speaking, such shape textures result from a compromise between minimizing a bending energy while rewarding an increase in interfacial area. Yet, we choose to treat the principal curvatures independently, which is not the case in their model.

Outline. In Sect. 2, we introduce the Willmore and the Helfrich energies, as well as their classical phase-field approximations in light of the $\Gamma$-convergence framework. In Sect. 3, we build the phase-field functional and justify its construction with heuristic arguments and in terms of $\Gamma$-limsup. Section 4 describes the computational framework and displays a large number of simulations. Finally, we discuss in Sect. 5 the implications of a unifying theory on applied fields and present future extensions to this work.

\section{Background}

We begin by introducing the Willmore and the Helfrich energies in more detail, then sketch the notion of $\Gamma$-convergence before moving on with their classical phase-field approximations.

But first, let us describe curvatures in simple terms. Curvature measures how much a line or a surface is locally deviating from a straight line or a flat plane. For a line contained in a plane, the curvature $\kappa$ at a point is the inverse $\frac{1}{r}$ of the radius of the osculating circle, i.e., the tangent circle that approaches the curves most tightly at this point. Surfaces are characterized at each point by two principal curvatures $\kappa_{1}$ and $\kappa_{2}$, that correspond to the maximal and minimal curvatures of the lines resulting from the perpendicular intersection of the surface with a plane. The maximally and minimally curved lines are directed by two perpendicu- 
lar principal directions, tangent to the surface. For instance, the principal curvatures are zero on a plane; on a sphere of radius $r$, they are all equal to $\left(\frac{1}{r}, \frac{1}{r}\right)$; on a cylinder of base radius $R$, they are all equal to $\left(\frac{1}{R}, 0\right)$.

\subsection{The Willmore, Helfrich, and Generalized Curvature Functionals}

In the 1970's, Canham [18], and subsequently Helfrich [44], proposed to model the surface of a biomembrane as a minimizer of a curvature bending energy, or Helfrich energy

$\mathbf{E}_{\mathrm{H}}(\mathscr{S})=\int_{\mathscr{S}}\left(\frac{\chi_{b}}{2}\left(H-H_{0}\right)^{2}+\chi_{G} K\right) \mathrm{d} A$.

In this expression, $\mathscr{S}$ is a smooth compact orientable surface in $\mathbb{R}^{3}$ whose principal curvatures are denoted $\kappa_{1}$ and $\kappa_{2}$, with the convention $\kappa_{1} \geq \kappa_{2}$. The mean curvature ${ }^{1} H=\kappa_{1}+\kappa_{2}$ and the Gaussian curvature $K=\kappa_{1} \kappa_{2}$ are respectively the sum and the product of the principal curvatures. The signs of $H, \kappa_{1}$, and $\kappa_{2}$ depend on the orientation of $\mathscr{S}$, while that of $K$ does not. Throughout this work, we use the convention that $H$ should be positive on convex objects like spheres. Let us remark that there is a closed relationship between $\left(\kappa_{1}, \kappa_{2}\right)$ and $(H, K)$, provided by the bijection $\phi(x, y)=(x+y, x y)$ from $\left\{(x, y) \in \mathbb{R}^{2} \mid y \leq x\right\}$ onto $\left\{(x, y) \in \mathbb{R}^{2} \mid y \leq \frac{1}{4} x^{2}\right\}$,

$$
\left\{\begin{array} { l } 
{ H = \kappa _ { 1 } + \kappa _ { 2 } } \\
{ K = \kappa _ { 1 } \kappa _ { 2 } } \\
{ \kappa _ { 1 } \geq \kappa _ { 2 } }
\end{array} \Leftrightarrow \left\{\begin{array}{l}
\kappa_{1}=\left(H+\sqrt{H^{2}-4 K}\right) / 2 \\
\kappa_{2}=\left(H-\sqrt{H^{2}-4 K}\right) / 2
\end{array}\right.\right.
$$

and that $H^{2}-4 K=\left(\kappa_{1}-\kappa_{2}\right)^{2}$.

In (3), the coefficients $\chi_{b}>0$ and $\chi_{G}$ are the bending and Gaussian rigidities. The parameter $H_{0}$, or spontaneous curvature, models the asymmetry of the two layers composing the bilipidic membrane [32,98]. The Willmore energy, defined as

$\mathbf{E}_{\mathrm{W}}(\mathscr{S})=\int_{\mathscr{S}} H^{2} \mathrm{~d} A$

is then a special case of the Helfrich energy, with $\chi_{G}=0$, $\chi_{b}=2$, and $H_{0}=0$.

In both of the classical functionals (3) and (5), the integrand is a polynomial $p\left(\kappa_{1}, \kappa_{2}\right)$ symmetric in the principal curvatures, i.e., $p\left(\kappa_{1}, \kappa_{2}\right)=p\left(\kappa_{2}, \kappa_{1}\right)$, since it can be reformulated as a polynomial of their sum $H$ and product $K$. However, one may want to construct a general curvature

\footnotetext{
1 The other convention is to define $H$ as the true mean of the curvatures, but we choose to keep consistency with the main references cited here, especially for the phase-field expressions presented later.
}

functional where $p$ is a smooth function with no symmetry constraint. A similar ${ }^{2}$ form is given in [43,106], for a smooth function $q(H, K)$. A generalization to functions that depend on the position and the normal to the surface can be found in $[19,26]$.

We will restrict ourselves to $p$ which are polynomials of degree 2, as the framework is then rich enough to generate complex shape textures. We are thus interested in the curvature functional

$$
\begin{aligned}
\mathbf{F}(\mathscr{S})= & \int_{\mathscr{S}}\left(a_{2,0} \kappa_{1}^{2}+a_{1,1} \kappa_{1} \kappa_{2}+a_{0,2} \kappa_{2}^{2}\right. \\
& \left.+a_{1,0} \kappa_{1}+a_{0,1} \kappa_{2}+a_{0,0}\right) d A \\
= & \int_{\mathscr{S}}\left(\sum_{|\alpha| \leq 2} a_{\alpha}\left(\kappa_{1}, \kappa_{2}\right)^{\alpha}\right) \mathrm{d} A,
\end{aligned}
$$

where we use multi-index notation.

\subsection{Phase-Fields and $\Gamma$-Convergence}

Numerically, critical points of the curvature energies (3), (5), and (6) can be searched for by following a gradient flow. Before implementing an algorithm, it is however preferable to convert these sharp-interface functionals defined for $2 \mathrm{D}$ surfaces, to diffuse approximations defined for scalar fields in a $3 \mathrm{D}$ volume. This way, surfaces are implicitly represented as level sets of the volumetric scalar field, which allows us to address topological changes encountered in the flow seamlessly; whereas in explicit methods, surfaces are tracked as a mesh that needs dynamic remeshing to avoid entanglement through topological transitions [80]. The gain is considerable in view of the high topological complexity of the targeted shape textures.

This leads us to consider a phase-field energy approximating the original geometric problem, for instance as in [2729] for the modeling of biomembranes. Phase-fields have been extensively used to model phase separation in binary mixtures, beginning with the Cahn-Hilliard (or GinzburgLandau) energy [16,34], that was subsequently reused in several other contexts [54,72]. These functions typically take values close to 1 and -1 inside and outside a region, with a smooth transition between the two phases at the interface. A parameter $\epsilon>0$ represents the thickness of phase transition at the interface.

The quality of a diffuse approximation with phase-fields is typically studied in the $\Gamma$-convergence framework $[3,12,24]$.

\footnotetext{
2 Yet, please note that $p$ being smooth in $\left(\kappa_{1}, \kappa_{2}\right)$ is not equivalent to $q$ being smooth in $(H, K)$, where $q=p \circ \phi^{-1}$. It can be checked by taking $q(H, K)=2 \kappa_{1}=H+\sqrt{H^{2}-4 K}$, which is not differentiable at points where $H^{2}=4 K$, i.e., when $\kappa_{1}=\kappa_{2}$. On a surface, this happens at umbilical points, e.g., everywhere on spheres.
} 
$\Gamma$-convergence expresses the convergence of minimization problems, so that, rather than solving a limit problem, we solve a sequence of approaching problems (or the reverse). The complete convergence consists in a $\Gamma$-limsup, which relies on a constructive proof, and a $\Gamma$-liminf, generally more difficult to prove.

Definition 1 ( $\Gamma$-convergence) Given $X$ a metric space, let $F$ and $F_{\epsilon}$ be functions from $X$ to $[-\infty,+\infty]$, where the $F_{\epsilon}$ are defined for $\epsilon>0$. We say that the sequence $\left(F_{\epsilon}\right)_{\epsilon>0}$ $\Gamma$-converges to $F$ as $\epsilon \rightarrow 0$, and write

$\left[\Gamma(X)-\lim _{\epsilon \rightarrow 0} F_{\epsilon}\right]=F$,

if the following two bounds hold for any $u \in X$ :

[ $\Gamma$-liminf] For every sequence $\left(u_{\epsilon}\right)$ such that $u_{\epsilon} \rightarrow u$ in $X$,

$\liminf _{\epsilon \rightarrow 0} F_{\epsilon}\left(u_{\epsilon}\right) \geq F(u)$.

[ $\Gamma$-limsup] There exists a sequence $\left(u_{\epsilon}\right)$, called recovery sequence, such that $u_{\epsilon} \rightarrow u$ in $X$ and

$\lim _{\epsilon \rightarrow 0} F_{\epsilon}\left(u_{\epsilon}\right)=F(u)$.

$\Gamma$-convergence is especially interesting due to the following fundamental result.

Theorem 1 Let $X$ be a metric space, and let $F=\Gamma(X)-$ $\lim _{\epsilon \rightarrow 0} F_{\epsilon}$. Suppose that the sequence $F_{\epsilon}: X \rightarrow[-\infty,+\infty]$ is equi-coercive, i.e., for all $t \in \mathbb{R}$ there exists a compact set $K_{t} \subset X$ such that $\left\{F_{\epsilon} \leq t\right\} \subset K_{t}$. Then $F$ admits a minimum and

$\min _{X} F=\lim _{\epsilon \rightarrow 0} \inf _{X} F_{\epsilon}$.

Furthermore, if $u_{\epsilon}$ minimizes $F_{\epsilon}$ over $X$, then every cluster point of $\left(u_{\epsilon}\right)$ minimizes $F$ over $X$.

This ensures not only the convergence of the minimal values, but also of the minimizers themselves, up to a subsequence.

\subsection{Phase-Field Approximations of the Area, the Willmore, and the Helfrich Functionals}

In this paragraph, we present three classical diffuse approximations that are important to our development. We fix some mathematical notations beforehand.

Notations. Let $\Omega$ denote an open bounded connected set in $\mathbb{R}^{3}$ with piecewise smooth boundary. The usual Sobolev spaces are denoted by

$W^{k, p}(\Omega)=\left\{u \in L^{p}(\Omega)\left|D^{\alpha} u \in L^{p}(\Omega), \forall\right| \alpha \mid \leq k\right\}$ and are the sets of functions $\mathrm{u}$ in $L^{p}(\Omega)$ whose mixed partial derivatives $D^{\alpha} u$ exist in the weak sense and are in $L^{p}(\Omega)$, up to $|\alpha| \leq k$. A function $u \in L^{1}(\Omega)$ is of bounded variation if the following quantity, denoted by $\int_{\Omega}|D u|$, is finite [4]:

$\sup \left\{\int_{\Omega} u(x) \nabla \cdot \phi(x) \mathrm{d} x \mid \phi \in C_{c}^{1}\left(\Omega, \mathbb{R}^{3}\right),\|\phi\|_{L^{\infty}(\Omega)} \leq 1\right\}<\infty$.

By $B V(\Omega,\{-1,1\})$, we refer to the set of $L^{1}$ functions $u$ : $\Omega \rightarrow\{-1,1\}$ of bounded variation. For a set $E \subset \Omega, \chi_{E}$ designates the characteristic function of $E$.

Let $\mathbf{e}$ be a fixed unit-norm vector in $\mathbb{R}^{3}$. We consider a symmetric double-well function $W(s)=\frac{1}{4}\left(1-s^{2}\right)^{2}$ that vanishes at -1 and +1 . Note that its derivative is $W^{\prime}(s)=$ $s^{3}-s$. Let $\sigma=\int_{-1}^{1} \sqrt{2 W(s)} d s=\frac{4}{3 \sqrt{2}}$ denote a constant that only depends on the double-well.

For a function $u$ twice (weakly) differentiable, we define the normal vector field

$\mathbf{n}_{u}= \begin{cases}\frac{\nabla u}{|\nabla u|} & \text { on the set }\{\nabla u \neq 0\} \\ \mathbf{e} & \text { elsewhere, }\end{cases}$

that has unit norm, and is orthogonal to the level sets of $u$. We introduce the matrix field

$\mathscr{M}_{u}^{\epsilon}=-\epsilon \operatorname{Hess} u+\frac{W^{\prime}(u)}{\epsilon} \mathbf{n}_{u} \otimes \mathbf{n}_{u}$,

whose trace is equal to

$\operatorname{Tr} \mathscr{M}_{u}^{\epsilon}=-\epsilon \Delta u+\frac{W^{\prime}(u)}{\epsilon}$.

The classical approximations. The Cahn-Hilliard phase-field energy is known to approximate the area (or perimeter) functional, which measures the total area of surfaces in the $3 \mathrm{D}$ space. More precisely, let us introduce

$\mathscr{A}_{\epsilon}(u)=\left\{\begin{array}{l}\int_{\Omega}\left(\frac{\epsilon}{2}|\nabla u|^{2}+\frac{W(u)}{\epsilon}\right) \mathrm{d} x \quad \text { if } u \in W^{1,2}(\Omega) \\ +\infty \quad \text { otherwise in } L^{1}(\Omega)\end{array}\right.$

and the area functional

$\mathbf{E}_{A}(u)=\left\{\begin{array}{l}\frac{1}{2} \int_{\Omega}|D u| \quad \text { if } u \in \mathrm{BV}(\Omega,\{-1,1\}) \\ +\infty \quad \text { otherwise in } L^{1}(\Omega)\end{array}\right.$.

Following a conjecture of De Giorgi, Modica and Mortola [73] proved the $\Gamma$-convergence

$\left[\Gamma\left(L^{1}(\Omega)\right)-\lim _{\epsilon \rightarrow 0} \mathscr{A}_{\epsilon}\right]=\sigma \mathbf{E}_{A}$. 
This means that, if $E \subset \Omega$ is such that $\mathscr{S}=\partial E \cap \Omega$ is smooth and of finite area, and setting $u=2 \chi_{E}-1 \in$ $B V(\Omega,\{-1,1\})$, then the $\Gamma$-limsup provides a sequence of functions $\left(u_{\epsilon}\right) \in W^{1,2}(\Omega)$ such that $u_{\epsilon} \rightarrow u$ in $L^{1}(\Omega)$ and $\mathscr{A}_{\epsilon}\left(u_{\epsilon}\right) \rightarrow \sigma$ area $(\mathscr{S})$, i.e., their diffuse areas converge to the area of $\mathscr{S}$ up to a factor $\sigma$.

Subsequently, several authors $[7,9,74,78,91,105]$ studied diffuse approximations of the Willmore energy (5). Bellettini and Paolini [9] introduced the phase-field functionals

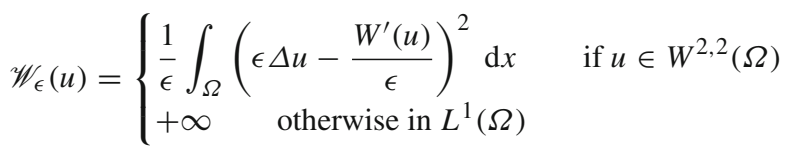

Note that the trace term $\operatorname{Tr} \mathscr{M}_{u}^{\epsilon}=-\epsilon \Delta u+\frac{W^{\prime}(u)}{\epsilon}$ inside the square is the $L^{2}$ gradient of $\frac{\epsilon}{2}|\nabla u|^{2}+\frac{W(u)}{\epsilon}$ which appears in the Cahn-Hilliard phase-field energy (8), in the same way as the mean curvature vector is the $L^{2}$ gradient of the area functional.

The $\Gamma$-limsup was showed in [9], using the same recovery sequence as for the area functional. The $\Gamma$-liminf was studied under several conditions in [7,74] and completed in [91]. Together with the $\Gamma$-limsup, this resulted in the $\Gamma$ convergence on smooth points of the form $u=2 \chi_{E}-1$ where $E \subset \Omega$ and $\partial E \cap \Omega$ is $C^{2}$ :

$$
\left[\Gamma\left(L^{1}(\Omega)\right)-\lim _{\epsilon \rightarrow 0} \mathscr{W}_{\epsilon}\right]\left(2 \chi_{E}-1\right)=\sigma \mathbf{E}_{\mathrm{W}}(\partial E \cap \Omega),
$$

but with the additional assumption that the diffuse surface areas $A_{\epsilon}$ remain uniformly bounded.

Finally, Bellettini and Mugnai [8] extended the Willmore phase-field functional to approximate the complete Helfrich energy with

$$
\begin{aligned}
& \mathscr{H}_{\epsilon}(u) \\
& =\left\{\begin{array}{c}
\int_{\Omega}\left[\frac{\chi_{b}}{2 \epsilon}\left(\operatorname{Tr} \mathscr{M}_{u}^{\epsilon}-\epsilon|\nabla u| H_{0}\right)^{2}\right. \\
\left.+\frac{\chi_{G}}{2 \epsilon}\left(\left(\operatorname{Tr} \mathscr{M}_{u}^{\epsilon}\right)^{2}-\left\|\mathscr{M}_{u}^{\epsilon}\right\|^{2}\right)\right] \mathrm{d} x \text { if } u \in C^{2}(\Omega), \\
+\infty \text { otherwise in } L^{1}(\Omega) .
\end{array}\right.
\end{aligned}
$$

Based on the previous results of Röger and Schätzle [91], and under the assumptions $H_{0}=0$ and $-\chi_{b}<\chi_{G}<0$, they showed that the $\Gamma$-convergence holds on smooth points $u=2 \chi_{E}-1$ where $E \subset \Omega$ is open and $\partial E \cap \Omega$ is $C^{2}$ :

$$
\left[\Gamma\left(L^{1}(\Omega)\right)-\lim _{\epsilon \rightarrow 0^{+}} \mathscr{H}_{\epsilon}\right]\left(2 \chi_{E}-1\right)=\sigma \mathbf{E}_{\mathrm{H}}(\partial E \cap \Omega),
$$

again using an additional uniform bound on the diffuse areas $A_{\epsilon}$.

Our aim is precisely to generalize the Helfrich phase-field formula $\mathscr{H}_{\epsilon}$ further to approximate the curvature functional (6), and provide a computational framework to simulate shape textures. We are now ready to construct a new phasefield functional, for which we will study the $\Gamma$-limsup property.

\section{Construction of the Phase-Field Functional}

In this section, we generalize the Helfrich phase-field energy $\mathscr{H}_{\epsilon}$ in (12) to approximate the functional $\mathbf{F}$ in (6), using the notations introduced in Sect. 2.3. We justify the construction with heuristic arguments, and show that the $\Gamma$-limsup is still satisfied, although we do not attempt to show the $\Gamma$-liminf.

\subsection{Diffuse Curvatures and Second Fundamental Form}

Let us notice that the diffuse expressions $\mathscr{W}_{\epsilon}$ and $\mathscr{H}_{\epsilon}$ in (11) and (12) both rely on the trace and the norm of the matrix field $\mathscr{M}_{u}^{\epsilon}$ introduced in (7), which is related to the second fundamental forms and the curvatures of the level sets of $u$ as follows. We define the diffuse second fundamental form $\mathscr{B}_{u}^{\epsilon}$ as well as the diffuse mean and Gaussian curvatures $\mathscr{H}_{u}^{\epsilon}$ and $\mathscr{K}_{u}^{\epsilon}$ using

$$
\begin{aligned}
\mathscr{B}_{u}^{\epsilon} & =\frac{\mathscr{M}_{u}^{\epsilon}}{\epsilon|\nabla u|} \\
\mathscr{H}_{u}^{\epsilon} & =\frac{\operatorname{Tr} \mathscr{M}_{u}^{\epsilon}}{\epsilon|\nabla u|} \\
\mathscr{K}_{u}^{\epsilon} & =\frac{1}{2 \epsilon^{2}|\nabla u|^{2}}\left[\left(\operatorname{Tr} \mathscr{M}_{u}^{\epsilon}\right)^{2}-\left\|\mathscr{M}_{u}^{\epsilon}\right\|^{2}\right]
\end{aligned}
$$

if $\nabla u \neq 0$, and zero otherwise. Informally, $\mathscr{B}_{u}^{\epsilon} \otimes \mathbf{n}_{u}$ at the point $x \in \Omega$ approximates the second fundamental form of the level surface $\{u=u(x)\}$ (well-defined if $\nabla u \neq 0$ on this set). $\mathscr{H}_{u} \mathbf{n}_{u}$ approximates the mean curvature vector, with the convention that it points inwards for convex sets, and $\mathscr{K}_{u}^{\epsilon}$ approximates the Gaussian curvature.

Based on the relations (4) linking $\left(\kappa_{1}, \kappa_{2}\right)$ to $(H, K)$, we also introduce the diffuse principal curvatures

$\begin{aligned} \kappa_{1, u}^{\epsilon} & =\frac{\mathscr{H}_{u}^{\epsilon}+\sqrt{\left(\left(\mathscr{H}_{u}^{\epsilon}\right)^{2}-4 \mathscr{K}_{u}^{\epsilon}\right)^{+}}}{2} \\ \kappa_{2, u}^{\epsilon}= & \frac{\mathscr{H}_{u}^{\epsilon}-\sqrt{\left(\left(\mathscr{H}_{u}^{\epsilon}\right)^{2}-4 \mathscr{K}_{u}^{\epsilon}\right)^{+}}}{2},\end{aligned}$

where we use the positive part $x^{+}=\max (0, x)$. 
It can be shown, using the implicit formulas summarized in [42], that the expressions from (14) to (18) coincide exactly with the second fundamental form and the respective curvatures of the level sets of $u$, in the special case where the function has a hyperbolic tangent profile

$u=\tanh \left(\frac{\operatorname{dist}_{\partial E}}{\sqrt{2} \epsilon}\right)$

where $E \subset \Omega$ is an open set with smooth boundary $\partial E \cap \Omega \in$ $\mathscr{C}^{2}$, and the signed distance from $\partial E$, denoted by $\operatorname{dist}_{\partial E}$, is by convention positive on $E$ and negative on $\Omega \backslash \bar{E}$. In diffuse approximations, the tanh profile is optimal ${ }^{3}$ and is generally used to construct the $\Gamma$-limsup recovery sequence.

The presence of the positive part in (17) and (18) ensures that the square root term is still defined when $\left(\mathscr{H}_{u}^{\epsilon}\right)^{2}-$ $4 \mathscr{K}_{u}^{\epsilon}<0$. This can happen, since for a general $u$, we have $\left(\mathscr{H}_{u}^{\epsilon}\right)^{2}-4 \mathscr{K}_{u}^{\epsilon}=\frac{2\left\|\mathscr{M}_{u}^{\epsilon}\right\|^{2}-\left(\operatorname{Tr} \mathscr{M}_{u}^{\epsilon}\right)^{2}}{\epsilon^{2}|\nabla u|^{2}}$, and the numerator is $a^{2}+b^{2}+c^{2}-2(a b+b c+a c)$ which possibly has negative values, where $a, b, c$ are the real eigenvalues of $\mathscr{M}_{u}^{\epsilon}$. However, if one of them is 0 , the numerator is a squared difference and the positive part is not useful. This is the case in particular for functions with tanh profile.

The expressions (17) and (18) can be reformulated with $\mathscr{M}_{u}^{\epsilon}$, by writing

$\begin{aligned} \kappa_{1, u}^{\epsilon} & =\frac{\operatorname{Tr} \mathscr{M}_{u}^{\epsilon}+\sqrt{\left(2\left\|\mathscr{M}_{u}^{\epsilon}\right\|^{2}-\left(\operatorname{Tr} \mathscr{M}_{u}^{\epsilon}\right)^{2}\right)^{+}}}{2 \epsilon|\nabla u|} \\ \kappa_{2, u}^{\epsilon} & =\frac{\operatorname{Tr} \mathscr{M}_{u}^{\epsilon}-\sqrt{\left(2\left\|\mathscr{M}_{u}^{\epsilon}\right\|^{2}-\left(\operatorname{Tr} \mathscr{M}_{u}^{\epsilon}\right)^{2}\right)^{+}}}{2 \epsilon|\nabla u|},\end{aligned}$

if $\nabla u \neq 0$, and zero otherwise. Note that $\mathscr{H}_{u}^{\epsilon}$ can be retrieved from their sum

$\kappa_{1, u}^{\epsilon}+\kappa_{2, u}^{\epsilon}=\mathscr{H}_{u}^{\epsilon}$

contrarily to $\mathscr{K}_{u}^{\epsilon}$ since their product is

$\kappa_{1, u}^{\epsilon} \kappa_{2, u}^{\epsilon}=\frac{1}{4}\left[\left(\mathscr{H}_{u}^{\epsilon}\right)^{2}-\left(\left(\mathscr{H}_{u}^{\epsilon}\right)^{2}-4 \mathscr{K}_{u}^{\epsilon}\right)^{+}\right] \leq \mathscr{K}_{u}^{\epsilon}$.

\subsection{Expression of the Phase-Field Functional}

Let $\mathbf{a}=\left(a_{2,0}, a_{1,1}, a_{0,2}, a_{1,0}, a_{0,1}, a_{0,0}\right)=\left(a_{\alpha}\right)_{|\alpha| \leq 2} \in$ $\mathbb{R}^{6}$ be a vector of real coefficients. The associated polynomial function is denoted by $p(x, y)=\sum_{|\alpha| \leq 2} a_{\alpha}(x, y)^{\alpha}$,

\footnotetext{
${ }^{3}$ It is the unique minimizer of the $1 \mathrm{D}$ version of the Cahn-Hilliard energy among increasing functions with limits \pm 1 at $\pm \infty$.
}

so that $\mathbf{F}(\mathscr{S})=\int_{\mathscr{S}} p\left(\kappa_{1}, \kappa_{2}\right) \mathrm{d} A$. Consider the following expression,

$\mathscr{E}_{\epsilon}(u)=\int_{\Omega} p\left(\kappa_{1, u}^{\epsilon}, \kappa_{2, u}^{\epsilon}\right) \epsilon|\nabla u|^{2} \mathrm{~d} x$

The heuristic intuition behind is that, if $u$ has a tanh profile with transition parameter $\epsilon$ as in (19), we can apply the coarea formula to obtain

$$
\begin{aligned}
& \int_{-1}^{1}\left(\int_{\{u=t\}} p\left(\kappa_{1, u}^{\epsilon}, \kappa_{2, u}^{\epsilon}\right) \epsilon|\nabla u| d \mathscr{H}^{2}\right) \mathrm{d} t \\
& =\int_{-1}^{1} \sqrt{2 W(t)} \mathbf{F}(\{u=t\}) \mathrm{d} t,
\end{aligned}
$$

where we use $|\nabla u|=\frac{\left(1-u^{2}\right)}{\sqrt{2} \epsilon} \neq 0$ and $\frac{1-t^{2}}{\sqrt{2}}=\sqrt{2 W(t)}$. This amounts to integrating the curvature functional $\mathbf{F}$ over all the level surfaces $\mathscr{S}_{t}=\{u=t\}$ of the phase-field $u$, appropriately weighted so that the largest contributions are given by level sets close to $\{u=0\}$. As $\epsilon$ goes to zero, the level sets $\{u=t\}$ concentrate around $\partial E$.

Still under the ansatz of tanh profile, $\mathscr{E}_{\epsilon}$ can be developed in terms of the matrix field $\mathscr{M}_{u}^{\epsilon}$ :

$$
\begin{aligned}
\widetilde{\mathscr{E}}_{\epsilon}(u)= & \int_{\Omega}\left[\frac{a_{2,0}+a_{0,2}-a_{1,1}}{4 \epsilon}\left(2\left\|\mathscr{M}_{u}^{\epsilon}\right\|^{2}-\left(\operatorname{Tr} \mathscr{M}_{u}^{\epsilon}\right)^{2}\right)^{+}\right. \\
& +\frac{a_{2,0}+a_{0,2}+a_{1,1}}{4 \epsilon}\left(\operatorname{Tr} \mathscr{M}_{u}^{\epsilon}\right)^{2} \\
& +\frac{a_{2,0}-a_{0,2}}{2 \epsilon} \operatorname{Tr} \mathscr{M}_{u}^{\epsilon} \sqrt{\left(2\left\|\mathscr{M}_{u}^{\epsilon}\right\|^{2}-\left(\operatorname{Tr} \mathscr{M}_{u}^{\epsilon}\right)^{2}\right)^{+}} \\
& +\frac{a_{1,0}+a_{0,1}}{2}|\nabla u| \operatorname{Tr} \mathscr{M}_{u}^{\epsilon} \\
& +\frac{a_{1,0}-a_{0,1}}{2}|\nabla u| \sqrt{\left(2\left\|\mathscr{M}_{u}^{\epsilon}\right\|^{2}-\left(\operatorname{Tr} \mathscr{M}_{u}^{\epsilon}\right)^{2}\right)^{+}} \\
& \left.+a_{0,0} \epsilon|\nabla u|^{2}\right] \mathrm{d} x .
\end{aligned}
$$

For a general $u \in W^{2,2}(\Omega)$, the expressions of $\mathscr{E}_{\epsilon}(u)$ and $\widetilde{\mathscr{E}}_{\epsilon}(u)$ coincide if $u$ is such that

$\mathscr{L}^{3}\left(\{|\nabla u|=0\} \cap\left\{\mathscr{M}_{u}^{\epsilon} \neq 0\right\}\right)=0$,

which is satisfied for functions with tanh profile.

Finally, $\widetilde{\mathscr{E}}_{\epsilon}(u)$ can be simplified further, by replacing the positive part in the first term outside the square root directly by $2\left\|\mathscr{M}_{u}^{\epsilon}\right\|^{2}-\left(\operatorname{Tr} \mathscr{M}_{u}^{\epsilon}\right)^{2}$. As said earlier, this is true of the special tanh case, where $2\left\|\mathscr{M}_{u}^{\epsilon}\right\|^{2}-\left(\operatorname{Tr} \mathscr{M}_{u}^{\epsilon}\right)^{2} \geq 0$ is $(a-b)^{2}$, where $a, b$ and 0 are the eigenvalues of $\mathscr{M}_{u}^{\epsilon}$. This leads to the final form, defined for any $u \in W^{2,2}(\Omega)$,

$$
\mathscr{F}_{\epsilon}(u)=\int_{\Omega}\left[\frac{a_{2,0}+a_{0,2}-a_{1,1}}{2 \epsilon}\left\|\mathscr{M}_{u}^{\epsilon}\right\|^{2}+\frac{a_{1,1}}{2 \epsilon}\left(\operatorname{Tr} \mathscr{M}_{u}^{\epsilon}\right)^{2}\right.
$$




$$
\begin{aligned}
& +\frac{a_{2,0}-a_{0,2}}{2 \epsilon} \operatorname{Tr} \mathscr{M}_{u}^{\epsilon} \sqrt{\left(2\left\|\mathscr{M}_{u}^{\epsilon}\right\|^{2}-\left(\operatorname{Tr} \mathscr{M}_{u}^{\epsilon}\right)^{2}\right)^{+}} \\
& +\frac{a_{1,0}+a_{0,1}}{2}|\nabla u| \operatorname{Tr} \mathscr{M}_{u}^{\epsilon} \\
& +\frac{a_{1,0}-a_{0,1}}{2}|\nabla u| \sqrt{\left(2\left\|\mathscr{M}_{u}^{\epsilon}\right\|^{2}-\left(\operatorname{Tr} \mathscr{M}_{u}^{\epsilon}\right)^{2}\right)^{+}} \\
& \left.+a_{0,0} \epsilon|\nabla u|^{2}\right] \mathrm{d} x .
\end{aligned}
$$

This phase-field functional is devised to be a diffuse approximation of the sharp-interface functional $\mathbf{F}$ (6), up to the multiplicative factor $\sigma$.

Comparison with the Willmore and the Helfrich diffuse approximations. It can be checked that the proposed formulation $\mathscr{F}_{\epsilon}$ is indeed a generalization of the previous formulas $\mathscr{W}_{\epsilon}$ and $\mathscr{H}_{\epsilon}$, by specifying the polynomial coefficients of the Willmore energy,

$p\left(\kappa_{1}, \kappa_{2}\right)=H^{2} \quad$ with $\quad \mathbf{a}=(1,2,1,0,0,0)$,

and of the Helfrich energy,

$$
\begin{aligned}
& p\left(\kappa_{1}, \kappa_{2}\right)=\frac{\chi_{b}}{2}\left(H-H_{0}\right)^{2}+\chi_{G} K \quad \text { with } \\
& \quad \mathbf{a}=\left(\frac{\chi_{b}}{2}, \chi_{b}+\chi_{G}, \frac{\chi_{b}}{2},-\chi_{b} H_{0},-\chi_{b} H_{0}, \frac{\chi_{b}}{2} H_{0}^{2}\right) .
\end{aligned}
$$

\section{3 Г-Limsup Property}

As explained in Sect. 2.2, the approximation of the target functional $\mathbf{F}$ by the sequence $\left(\mathscr{F}_{\epsilon}\right)_{\epsilon>0}$ can be studied in the $\Gamma$-convergence framework. Here, we assert that the phasefield functional $\mathscr{F}_{\epsilon}$ satisfies the $\Gamma$-limsup property, thus extending the result of [8].

Theorem 2 ( $\Gamma$-limsup inequality) Let $E \subset \Omega$ be $a$ bounded open set, such that $\partial E \cap \Omega$ is of class $\mathscr{C}^{2}$. The functionals $\mathscr{F}_{\epsilon}$ and $\mathbf{F}$ are defined as in (20) and (6). Then there exists a sequence of functionals $\left(u_{\epsilon}\right)_{\epsilon>0} \subset W^{2,2}(\Omega)$ such that

$$
\begin{aligned}
& \lim _{\epsilon \rightarrow 0^{+}} u_{\epsilon}=2 \chi_{E}-1 \text { in } L^{1}(\Omega), \\
& \lim _{\epsilon \rightarrow 0^{+}} \epsilon\left|\nabla u_{\epsilon}\right|^{2} \mathscr{L}_{\mid \Omega}^{3}=\sigma \mathscr{H}_{\mid \partial E}^{2} \text { as Radon measures, } \\
& \lim _{\epsilon \rightarrow 0^{+}} \mathscr{F} \epsilon\left(u_{\epsilon}\right)=\sigma \mathbf{F}(E)
\end{aligned}
$$

The proof is given in the Appendix. It consists in showing that the recovery sequence constructed in [8] still satisfies the theorem for our more general formulation $\mathscr{F}_{\epsilon}$. The first and third properties correspond to the existence of a recovery sequence. The second property loosely means that the measure whose density is $\epsilon\left|\nabla u_{\epsilon}\right|^{2}$ in the 3D volumetric space concentrates into the measure induced by the area on the 2D surface. This intuition is in accordance with the way we constructed $\mathscr{F}_{\epsilon}$ (see beginning of Sect. 3.2).
However, whether or not the $\Gamma$-liminf holds still remains an open question, except in the special cases of the Willmore and the Helfrich energies with additional assumptions, as seen in Sect. 2.3. Yet, we believe that, even if the $\Gamma$ convergence could fail in general, this does not constitute a serious impediment to our phase-field expression being still of interest for generating shape textures.

\section{Simulations}

In this section, we demonstrate that the phase-field model $\mathscr{F}_{\epsilon}$ constructed in the previous section can generate a large range of shape textures (up to a slight regularization; see Sect. 2 of the Online Supplement). We describe curvatubes in Algorithm 1, and then show the results of four numerical experiments. The first one validates the approach by finding well-known Willmore minimizers. We then display a gallery of 10 shape textures. The effect of smoothly varying the generation parameters is shown in the third experiment, with a bilinear interpolation between 4 shape textures, layers, spheres, tubes, and sponges. Finally, 1000 shape textures are generated with random parameters and visualized in an atlas with UMAP. The numerical codes are fully available at

https://github.com/annasongmaths/curvatubes.

More details on the computation of the phase-field energy can be found in Sect. 2 of the Online Supplement.

\subsection{Curvatubes}

Shape textures are generated by minimizing the phase-field energy $\mathscr{F}_{\epsilon}(20)$ under a constraint of constant volume, with periodic boundary conditions. More exactly, given a random initialization of the phase-field $u$, we find a point of convergence with low energy of the so-called $H^{-1}$ flow $^{4}$

$\dot{u}=\Delta \frac{\partial \mathscr{F}_{\epsilon}}{\partial u}(u)$.

The $H^{-1}$ flow is mass-preserving, i.e., keeps constant the mass of $u$, denoted by $\bar{u}:=\frac{1}{\Omega} \int_{\Omega} u \mathrm{~d} x$. The preservation of mass approximately encodes a constraint of constant volume on the region enclosed by the surface $\{u=0\}$, if the phasefield $u \simeq \pm 1$ is nearly constant inside and outside.

The $H^{-1}$ flow can actually be expressed as a standard $L^{2}$ flow, by relying on the change of variable

$u=\nabla \cdot A+m_{0}$,

4 This is a gradient flow with respect to the $H^{-1}(\Omega)$ metric, where $H^{-1}(\Omega)$ is the dual of the space $H_{0}^{1}(\Omega)$, the closure of the set $C_{c}^{\infty}(\Omega)$ of smooth compactly-supported functions in $W^{1,2}(\Omega)[22,35]$. 
where $A: \Omega \rightarrow \mathbb{R}^{3}$ is a periodic vector field, and $m_{0} \in \mathbb{R}$ is the desired value of the average $\bar{u}$. We then define an energy with respect to $A$,

$G_{\epsilon}(A)=\mathscr{F}_{\epsilon}\left(\nabla \cdot A+m_{0}\right)$.

It can be checked that

$\frac{\partial G_{\epsilon}}{\partial A}(A)=-\nabla \frac{\partial \mathscr{F}_{\epsilon}}{\partial u}(u)$,

in such a way that a $L^{2}$ flow on $A$ becomes a $H^{-1}$ flow on $u$ :

$\dot{A}=-\frac{\partial G_{\epsilon}}{\partial A}(A) \Rightarrow \dot{u}=\Delta \frac{\partial \mathscr{F} \epsilon}{\partial u}(u)$

(provided that the derivatives in time and space of $A$ commute). Therefore, the $H^{-1}$ flow on $u$ starting at $u_{0}=$ $\nabla \cdot A_{0}+m_{0}$ can be solved as a usual $L^{2}$ flow on $A$.

To generate shape textures, the variable $A$ is initialized as a random white noise vector field $A_{0}$ and we reach a point of convergence of the $L^{2}$ flow $\dot{A}=-\frac{\partial G_{\epsilon}}{\partial A}$ with Adam [55,66], a gradient-based stochastic optimization algorithm. The change of variable (24) allows us to benefit from the computation of the $L^{2}$ gradient $\frac{\partial G_{\epsilon}}{\partial A}$ by the automatic differentiation engine provided by PyTorch [83], combined with the efficiency of Adam.

The generation model is summarized in Algorithm 1. It takes as inputs the initialization $A_{0}$, the coefficients $\mathbf{a}$ and the mass $m_{0}$. After convergence, the output shape texture is defined as the level surface $\{u=0\}$ of the final phase-field $u=\nabla \cdot A+m_{0}$. We color it in beige, and show the level set $\{u=0.05\}$ in dark red to enhance the visualization. An example of flow is given in Fig. 2, with the corresponding loss curves in Fig. 3.

Implementation details. The domain $\Omega$ is assimilated to a grid of size $100 \times 100 \times 100$ pixels with a fixed sampling step $\Delta x=0.01$. We take $\epsilon=0.02$, unless specified otherwise. The phase-field $u$ and the vector field $A$ are encoded as matrices whose coefficients specify the sampled values.

The discrete energies $\mathscr{F}_{\epsilon}$ (resp. $G_{\epsilon}$ ) are symbolically defined by a succession of elementary operations on $u$ (resp. A), before being differentiated automatically by PyTorch. In particular, the integral is encoded as a finite sum, while the differential operations $\nabla u$, Hess $u$, and $\nabla \cdot A$, are computed as classical finite differences that take into account the periodicity of the problem. To prevent the formation of artifacts, we apply a Gaussian blur $k$ with a small deviation (typically $\sigma_{k}=$ 2 pixels) to the phase-field $u$, before computing the finite differences. The norm of the gradient $|\nabla u|$ is modified by a small offset $\xi=10^{-6}$, as in $\sqrt{|\nabla u|^{2}+\xi^{2}}$ or $1 / \sqrt{|\nabla u|^{2}+\xi^{2}}$, to prevent non-differentiability at zero and division by zero. The positive part function $x^{+}$appearing in $\mathscr{F}_{\epsilon}$ is approximated by a smooth function $x^{+} \simeq \xi \log \left(1+\mathrm{e}^{x / \xi}\right)$. In Sect. 2 of the Online Supplement, we prove the convergence of the numerical approximations of the energies and their $L^{2}$ gradients by their discrete counterparts (computed with finite differences and automatic differentiation) as the resolution of the grid tends to infinity.

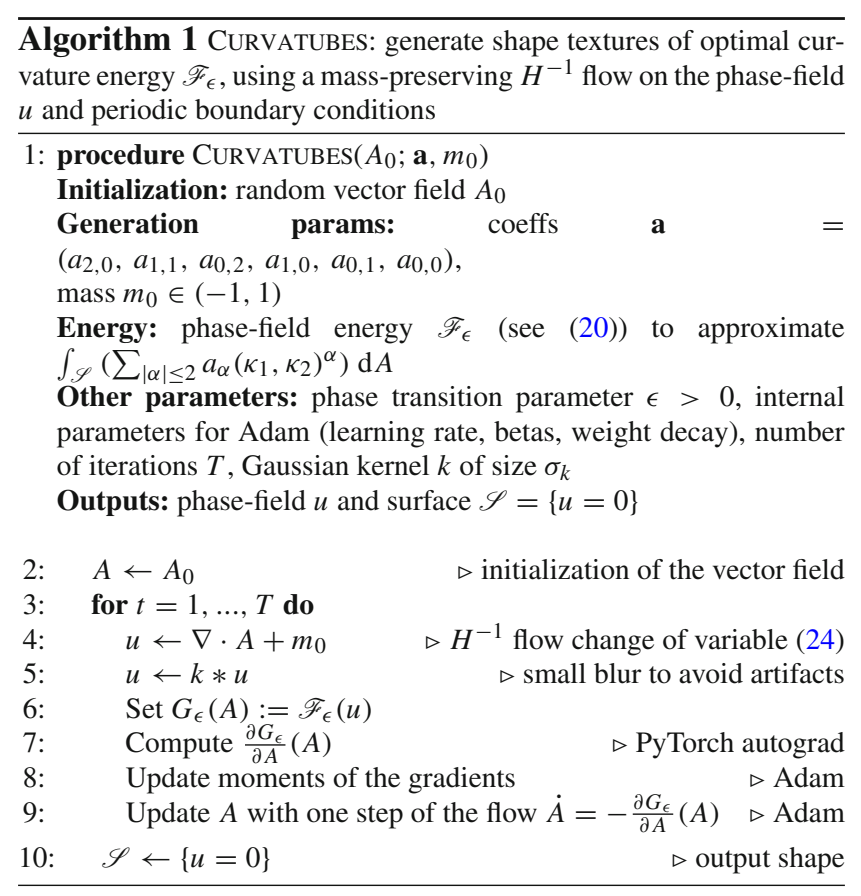

With Adam, the step size and direction at each point are computed in an adaptive way, by taking into account the past history of the gradients to estimate their first and second moments. In the simulations presented thereafter, Adam was run with a learning rate $l r=0.001$, betas $=(0.9,0.999)$, and no weight decay. We stopped the algorithm typically after 8000 iterations, as the convergence was estimated to be reached, which induced up to 150 seconds of computation time per shape with a simulation domain of size $100 \times 100 \times 100$ pixels.

Generation parameters, shape textures, curvature diagrams. The generation of shape textures relies on the principle that a generation parameter vector $\left(\mathbf{a}, m_{0}\right)$ should consistently correspond to a single shape texture, across different white noise initializations of $A_{0}$. We "measure" the texture of a shape defined by a surface $\mathscr{S}$ through its curvature diagram, which represents the distribution of the curvatures $\left(\kappa_{1}, \kappa_{2}\right)$ on $\mathscr{S}$ (see bottom row of Fig. 2 for instance). More precisely, we are interested in the law of the random variable $\left(\kappa_{1}, \kappa_{2}\right)$ defined by

$$
\begin{aligned}
\mathbb{P}\left[\left(\kappa_{1}, \kappa_{2}\right) \in \mathscr{B}\right] & =\frac{1}{\operatorname{Area}(\mathscr{S})} \int_{\mathscr{S}} \chi_{\left(\kappa_{1}, \kappa_{2}\right) \in \mathscr{B}} d \mathscr{H}^{2} \\
& =\frac{\operatorname{Area}\left(\left\{x \in \mathscr{S} \mid\left(\kappa_{1}, \kappa_{2}\right)(x) \in \mathscr{B}\right\}\right)}{\operatorname{Area}(\mathscr{S})},
\end{aligned}
$$

for a Borel set $\mathscr{B}$ of $\mathbb{R}^{2}$. 

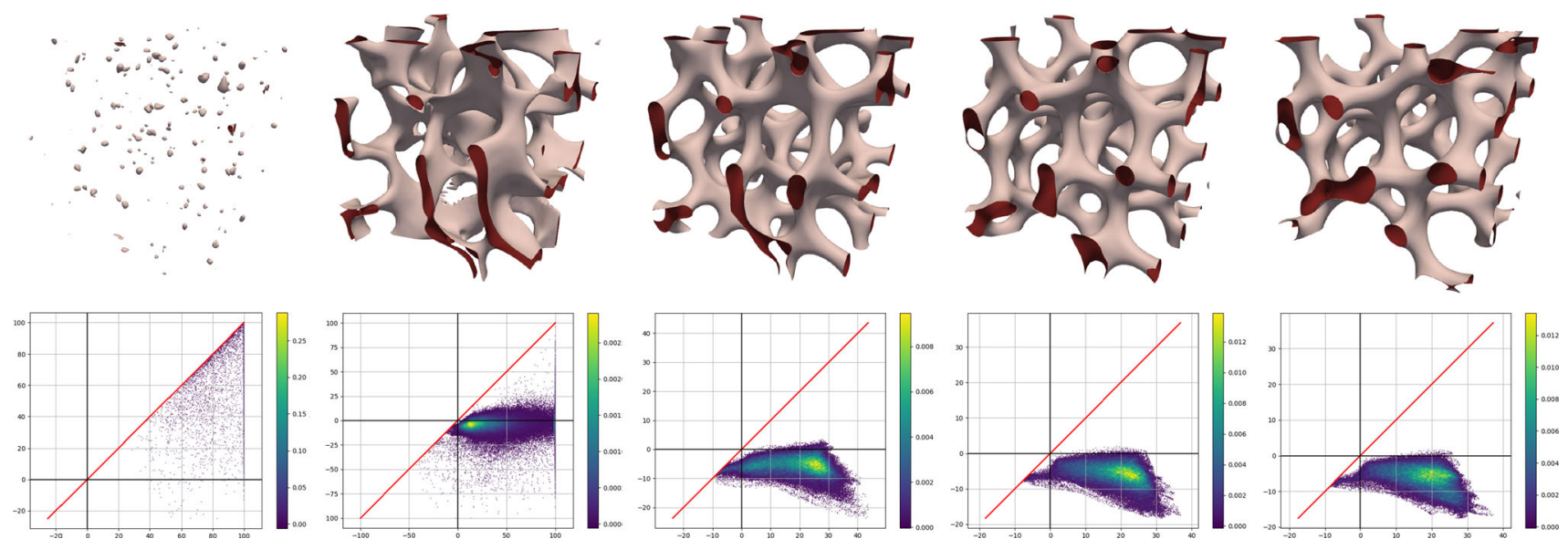

Fig. 2 Evolution of the zero level set of $u$ during the $H^{-1}$ flow, pictured at iterations $10,300,1000,4000,8000$, with the generation parameters $\mathbf{a}=(1,2.4,9,30,170,-195)$ and $m_{0}=-0.66$. Top row: the shape textures. Bottom row: their corresponding curvature diagrams. In the early iterations, the zero level set is not smoothly defined and only few values of $u$ are above zero, but their average remains $m_{0}$
The curvature diagram is an indicator of the local behavior of the surface. For a perfect sphere of radius $R$, it should be a unit Dirac mass $\delta_{\left(\frac{1}{R}, \frac{1}{R}\right)}$ sitting on the $\{y=x, x>0\}$ half-diagonal, since $\kappa_{1}=\kappa_{2}=\frac{1}{R}$ everywhere on a sphere. Likewise, a cylinder of base radius $r$ should have its diagram reduced to $\delta_{\left(\frac{1}{r}, 0\right)}$ on the horizontal half-line $\{y=0, x>0\}$; a plane would be represented as $\delta_{(0,0)}$; finally, a Dirac mass such as $\delta_{(1,-1)}$ should correspond to a sponge-like shape. Note that, per definition of the curvatures $\kappa_{1} \geq \kappa_{2}$, the distribution is contained in the lower mid-plane $\{y \leq x\}$.

To obtain a curvature diagram, we first extract the 2D mesh of the surface $\mathscr{S}=\{u=0\}$ from the 3D volume $u$, by using the marching cubes algorithm [63]. Then, the diffuse curvatures $\left(\kappa_{1, u}^{\epsilon}, \kappa_{2, u}^{\epsilon}\right)$ (see (17) and (18)) are interpolated at the barycenter of each cell of the mesh. Their values are of importance proportional to the area of the cell, resulting in a weighted point cloud

$$
\sum_{\text {cells } c} \operatorname{Area}[c] \delta_{\left(\kappa_{1, u}^{\epsilon}, \kappa_{2, u}^{\epsilon}\right)}[c]
$$

which we plot in the curvature diagram. Note that the diffuse curvatures coincide with the curvatures of the levels sets for functions with tanh profile (19), but are only approximations in the general case. Hence, the curvature diagrams shown throughout this article are only approximations of the exact curvature diagrams, most precise for those $u$ having nearly a tanh profile..$^{5}$

In our simulations, the shapes are rarely perfectly spherical, cylindrical, or flat, so that the distribution is dispersed rather than concentrated into a single Dirac mass. To ease the

\footnotetext{
5 The deviation of the phase-fields $u$ from the tanh profile is measured by the normalized discrepancy, see (27) in Experiment 4.
}
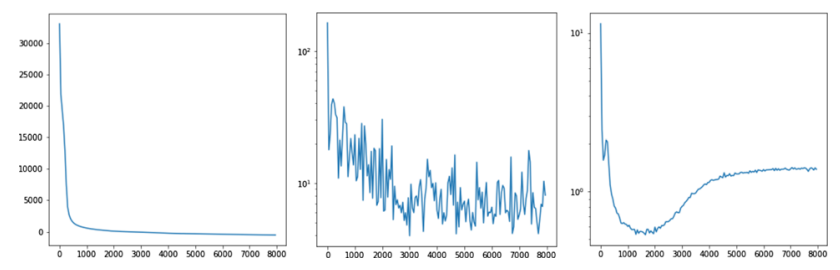

Fig. 3 Corresponding loss curves (see Fig. 2). From left to right: the loss $\mathscr{F}_{\epsilon}(u)$, the maximal value of $\left|\frac{\partial G_{\epsilon}}{\partial A}\right|$ and the average $\frac{1}{|\Omega|} \int_{\Omega}\left|\frac{\partial G_{\epsilon}}{\partial A}\right|$, as $u$ evolves along the iterations

visualization of the curvature diagrams, the identity diagonal $\{y=x\}$ is enhanced as a red line and the values truncated between -100 and 100 .

We compare curvature diagrams with each other using the Wasserstein (or Earth Mover's) distance. We approximate this quantity with the regularized Sinkhorn algorithm of the geomloss module [38], with the parameters $p=2$, blur $=$ 1 and reach $=20$. As detailed in [37], these correspond to the resolution of an unbalanced transport problem [102] using a ground cost function of $\mathrm{C}(x, y)=\frac{1}{2}\|x-y\|^{2}$, with a transport plan that is blurred at resolution of 1 and with a maximum transport distance of the order of 20.

Figure 2 shows that the curvature diagrams of the evolving level surface also converge towards a final diagram. In Fig. 4, we check that for a single generation parameter value $\left(\mathbf{a}, m_{0}\right), 5$ different initializations still give similar curvature diagrams. We found that the mean pairwise Wasserstein distance between different curvature diagrams was around 1.32 for these 5 shapes, i.e. only $10.7 \%$ of the mean pairwise distance 12.35 measured between the curvature diagrams of 1000 random shapes (see Experiment 4). Due to the high dimensionality of the space of curvature diagrams endowed with the Wasserstein metric, this ratio actually indicates a 

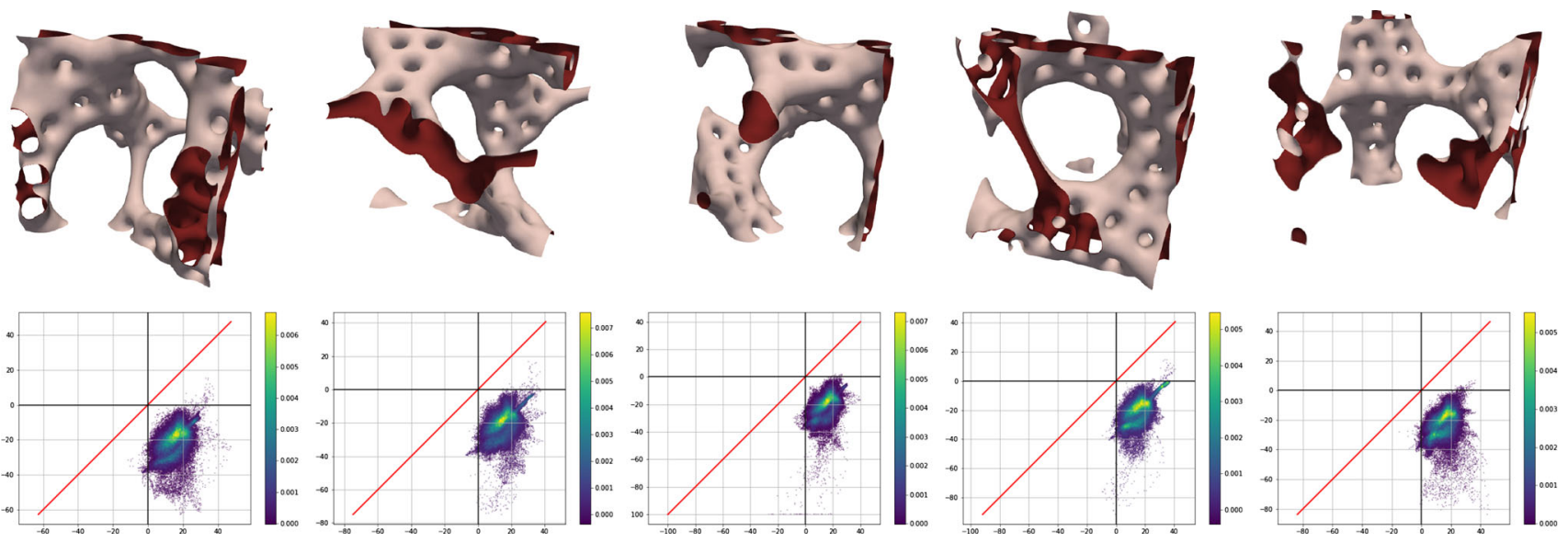

Fig. 4 Same generation parameters, different initializations. Using the same generation parameters $\mathbf{a}=(1,-0.35,1.02,-40,100,1600)$ and $m_{0}=-0.69$, we start from five random initializations for $A_{0}$. Top row: the shape textures. Bottom row: their corresponding curvature diagrams. The five shapes are visually similar at a mesoscopic scale, but dissimilar at the macroscopic scale. The diagrams and their pairwise Wasserstein distances show that the curvature statistics are not changing much when feeding the same generation parameters to the algorithm. Texture in shapes hence seems to be well captured by curvature diagrams, and to be consistent with the generation parameters at a mesoscopic scale good clustering of these 5 shapes. Indeed, as dimension increases, points easily become sparser: the distance to the farthest neighbor becomes comparable to that to the nearest one due to the "curse of dimensionality" [2,11]. After embedding the combined dataset of $1000+5$ shapes in 2D with UMAP, similarly to Experiment 4, the 5 shapes are more obviously clustered in low dimension (Online Supplement, Figure 1), since the new pairwise Euclidean distance within them was only 0.115 , i.e. only $2.67 \%$ compared to 4.3 for the other 1000 shapes.

Therefore, curvature diagrams and generation parameters seem to capture well the notion of shape texture.

\subsection{Experiment 1: Validation with the Willmore Flow and Known Minimizers of Genus 0, 1, and 2}

The algorithmic framework, which combines automatic differentiation and control of gradient flows by external optimizers, is validated in the fundamental special case of the $L^{2}$ Willmore flow. This flow is computed using Algorithm 2, with the parameters $\mathbf{a}=(1,2,1,0,0,0)$, and with replicate boundary conditions (which corresponds to the assumption that the gradient $\nabla u$ is orthogonal to the domain boundary, i.e., $\nabla u \cdot \mathbf{n}_{\partial \Omega}=0$ ). Let us recall from Sect. 2.3 that the Willmore phase-field energy writes $\mathscr{F}_{\epsilon}=\frac{1}{\epsilon} \int_{\Omega}(\epsilon \Delta u-$ $\left.\frac{W^{\prime}(u)}{\epsilon}\right)^{2} \mathrm{~d} x$ and approximates $\sigma \int_{\mathscr{S}} H^{2} \mathrm{~d} A$. We numerically check that the simulated Willmore flow converges towards known global minimizers of fixed genus 0,1 , and 2, by initializing the flow near them. Here, the gradient descent is controlled by the L-BFGS optimizer, as it was empirically found to converge faster than Adam ${ }^{6}$. L-BFGS approximates the BFGS algorithm, a quasi-Newton method that combines a line search to an estimation of the Hessian of the loss [39,52].

Algorithm $2 L^{2}$ FLOW: follow the gradient flow of the phase-field energy $\mathscr{F}_{\epsilon}$ until convergence, with given initialization and replicate boundary conditions

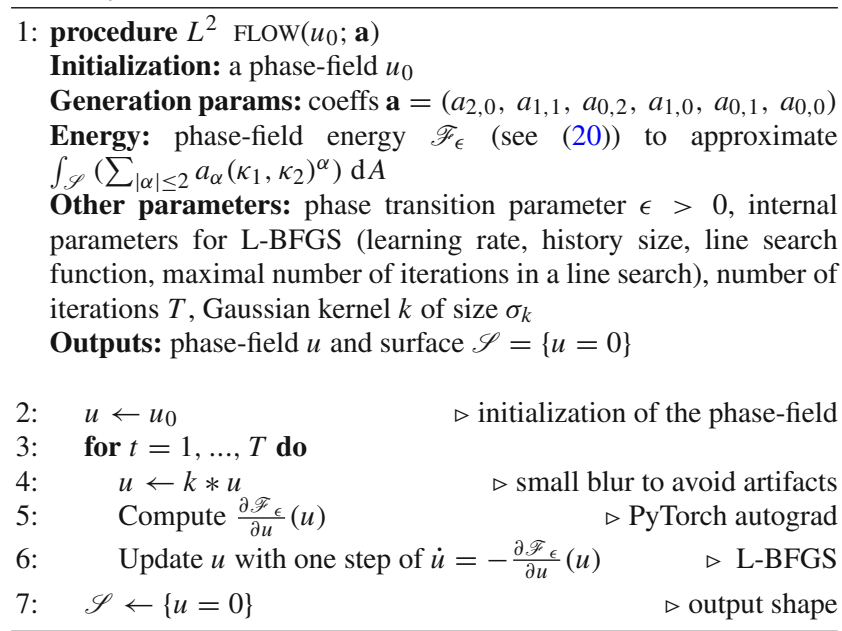

The Willmore minimizers of genus 0 are spheres of any radius [110], which achieve the minimal value ${ }^{7}$

$\int_{\mathscr{S}} H^{2} \mathrm{~d} A=\int_{\mathscr{S}}\left(\kappa_{1}+\kappa_{2}\right)^{2} \mathrm{~d} A=4 \times 4 \pi$.

\footnotetext{
6 This faster convergence holds for the Willmore flow, but during the $H^{-1}$ flow of more general energies, L-BFGS easily gets stuck at a point without any further progress (see Online Supplement, Figure 2).

7 We have to multiply the conventional values by 4 , as $H$ is defined as the (real) mean of the curvatures $\frac{\kappa_{1}+\kappa_{2}}{2}$ in other works.
} 


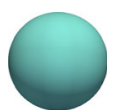

Fig. 5 Willmore minimizer of genus 0 . The flow is stationary on the sphere. The diffuse Willmore energy and the diffuse area are close to their mathematical values

For surfaces of genus 1, [67] proved that the minimal value $4 \times 2 \pi^{2}$ is achieved by the Clifford torus (up to conformal transformations), defined by a special ratio $\frac{1}{\sqrt{2}}$ between the radius of the generating circle and the distance to the axis of revolution. However, the proof for genus $\geq 2$ has still not been completed, although several conjectures have been proposed. It has been shown that the minimum Willmore energy among all (orientable closed) surfaces of genus $g$ is less than $4 \times 8 \pi$, and converges to this value as the genus $g \rightarrow \infty$ [61]. The Lawson surfaces have also been conjectured to be the minimizers for a given genus (up to conformal transformations) $[45,60]$.

We compare the final value $\frac{\mathscr{F}_{\epsilon}}{\sigma}$ to the minimal values mentioned above, where $\sigma=\frac{4}{3 \sqrt{2}}$ is the constant introduced in Sect. 2. We correctly find that the flow is stationary on spheres (see Fig. 5). The energy $\frac{\mathscr{F}_{\epsilon}}{\sigma}$ deviates from $4 \times 4 \pi$ with only $0.3 \%$ of relative error. We also check that the value of the Cahn-Hilliard energy $\int_{\Omega}\left(\frac{\epsilon}{2}|\nabla u|^{2}+\frac{W(u)}{\epsilon}\right) \mathrm{d} A$, divided by $\sigma$, is close to the area of the sphere, with $0.5 \%$ of relative error. When departing from a holed cube, the flow converges to a Clifford torus with a characterizing ratio close to $\frac{1}{\sqrt{2}}$ up to a relative error of $3 \%$. We also find $\frac{\mathscr{F}_{\epsilon}}{\sigma} \simeq 4 \times 2 \pi^{2}$ up to a relative error of $0.4 \%$. Finally, starting from two holed cubes glued together, the flow converges to a sur-
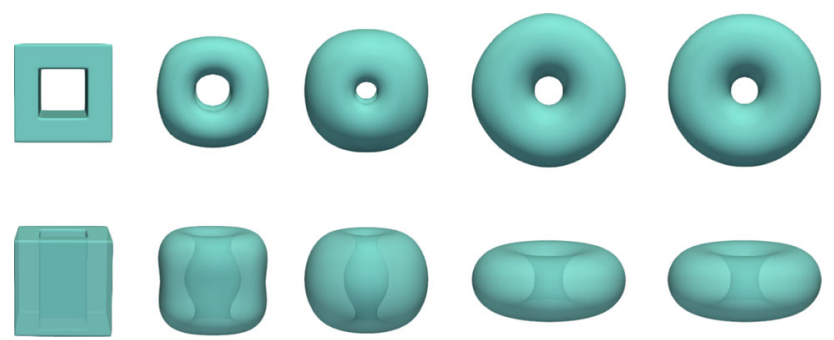

Fig. 6 Willmore flow towards minimizer of genus 1. The flow converges to a Clifford torus. The ratio of the torus and the diffuse Willmore energy are close to their mathematical values
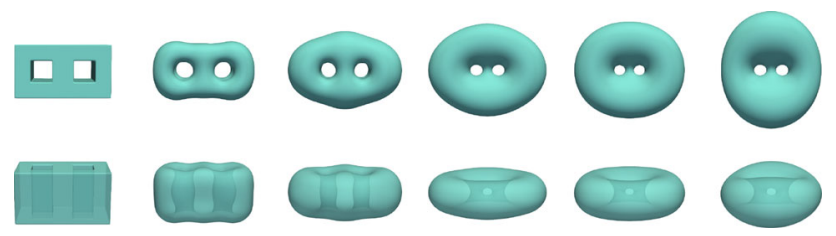

Fig. 7 Willmore flow towards minimizer of genus 2. The flow converges to a Lawson-like surface, as expected from conjectures face resembling a Lawson surface of genus 2, with minimal value $\frac{\mathscr{F}_{\epsilon}}{4 \sigma} \simeq 22.30$, which has a relative difference of $1.8 \%$ compared to the value found in [45]. In Figs. 5, 6, and 7, the parameters are $\epsilon=0.04, \mathbf{a}=(1,2,1,0,0,0)$ and $\sigma_{k}=1$ pixel. The grid size is $200 \times 200 \times 200$ in the first two simulations and $200 \times 300 \times 400$ in the third one. L-BFGS was run with a learning rate $l r=1$, a history size $h s=10$, and maximum 20 iterations in a line search.

\subsection{Experiment 2: A Gallery of Ten Shape Textures}

In Figs. 11 and 12, we show ten shape textures generated with Algorithm 1, and visualize the surfaces together with their curvature diagrams. The coefficients a and the mass $m_{0}=\frac{1}{|\Omega|} \int_{\Omega} u$ used for the simulations are specified in the tables. The mass is also expressed in percentage of volume enclosed by the surface compared to the total volume of the domain, approximated by $\frac{m_{0}+1}{2}$ since $u \simeq \pm 1$.

In practice, we find that the non-reduced polynomial expression

$h_{2}\left(H-H_{0}\right)^{2}+k_{1} K+\alpha\left(\kappa_{1}-\kappa_{1}^{0}\right)^{2}+\beta\left(\kappa_{2}-\kappa_{2}^{0}\right)^{2}$

induces a choice of parameters $\mathbf{b}=\left(h_{2}, H_{0}, k_{1}, \alpha, \kappa_{1}^{0}, \beta, \kappa_{2}^{0}\right)$ that are more interpretable than the coefficients $\mathbf{a}$ in the reduced form $\sum_{|\alpha| \leq 2} a_{\alpha}\left(\kappa_{1}, \kappa_{2}\right)^{\alpha}$. Using this formulation (26), it is easier to find reasonable values for which the energy leads to various tubular textures, without resulting in badlyconverged phase-fields (we have no theoretical justification to this yet, but this should be explored as a future work). Those phase-fields are often over-diffuse, have a high normalized discrepancy (defined in (27)), and do not present clear transitions between the phases \pm 1 . They may have no zero level set, may oscillate in space, which gives rise to pieces of surface concentrically enclosed in each other, or their zero level sets may be broken into irregular fragments of variable size (see Online Supplement, Figure 5). The shapes were hence generated either by choosing $\mathbf{b}$ manually, or by selecting a among random values from Experiment 4 that resulted in interesting shapes.

We found that curvatubes is able to generate very different shape textures (see Figs. 11 and 12, in which they are indexed by letters). Some shapes, such as (d), (e), (g), and (h) are smooth and spatially homogeneous in terms of visual aspect. Other shapes, such as (b), (c), (f), and especially (i), seem to possess a multi-scale texture or "metatexture" that makes them appear as spatially heterogeneous 
and anisotropic. The surface can be piecewise-smooth only, as in (c). Tubules are not necessarily smoothly turning cylinders, but can have some tortuosity as in (j). Finally, note that (h) combines flat regions and tubules, in a similar way to trabecular bone.

\subsection{Experiment 3: Bilinear Interpolation Between Four Shape Textures}

In Fig. 8, we illustrate how continuously varying the generation parameters $\left(\mathbf{a}, m_{0}\right)$ impacts on the morphologies, by interpolating the parameters of four shape textures: layers, spheres, tubes, and sponges. The respective values can be found in Table 1. All simulations are run by starting from the same initialization $A_{0}$.

We can see that the morphology is smoothly changing throughout the figure: for instance, the transition between spheres and tubes is characterized by tubes terminated on one side by end-caps, while layers increase in proportion compared to tubules when approaching the top left corner. The curvature diagrams are displayed in Fig. 9, and they are quite continuously evolving as we change the generation parameters. The four diagrams at the corners reflect well the typical curvature distribution of layers, spheres, tubes, and sponges, as expected (see Sect. 4.1).

\subsection{Experiment 4: Generation of 1000 Shapes Viewed in UMAP}

Our final experiment is designed to explore the space of possible shape textures with curvatubes and visualize them in a 2D atlas (see Fig. 10). The generation parameters were chosen randomly. We fixed $a_{2,0}=1$, and arbitrarily chose the other coefficients according to a uniform law in the following intervals: $a_{1,1} \in(-4,4), a_{0,2} \in(1 / 15,15), b_{1,0} \in$ (-200, 200), $b_{0,1} \in(-200,200), c \in(-3000,3000)$, and the mass $m_{0} \in(-0.75,-0.15)$ which represented from $12.5 \%$ to $42.5 \%$ of relative volume occupied by the phase $\{u \simeq 1\}$. The initialization $A_{0}$ was refreshed for each simulation.

By doing this, the algorithm was pushed to its limits, as some values of coefficients chosen in this random way led to an ill-posed geometric minimization problem. Yet, even in these cases, the algorithm did not diverge to $\mathrm{NaN}$ values, but the function $u$ simply did not converge to a phase-field with two distinct phases, or had no zero level set, or the zero level set was not smooth and was fragmented into pieces. To reject such situations, we gauged the viability of the parameters by computing the discrepancy $\left.\int_{\Omega}\left|\frac{\epsilon}{2}\right| \nabla u\right|^{2}-\frac{W(u)}{\epsilon} \mid \mathrm{d} x$ of the phase-field, normalized by the diffuse area, i.e., the Cahn-Hilliard energy. We call this quantity the normalized 


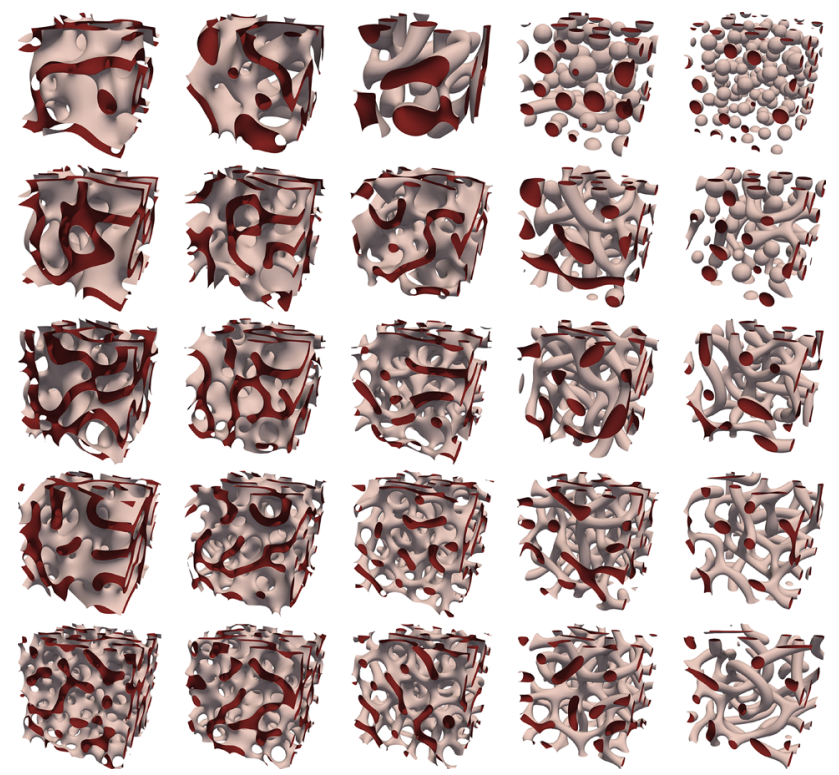

Fig. 8 Bilinear interpolation between layers, spheres, tubes, and sponges. Using the same initialization $A_{0}$ and interpolating between four reference generation parameters gives rise to a continuum of shape textures

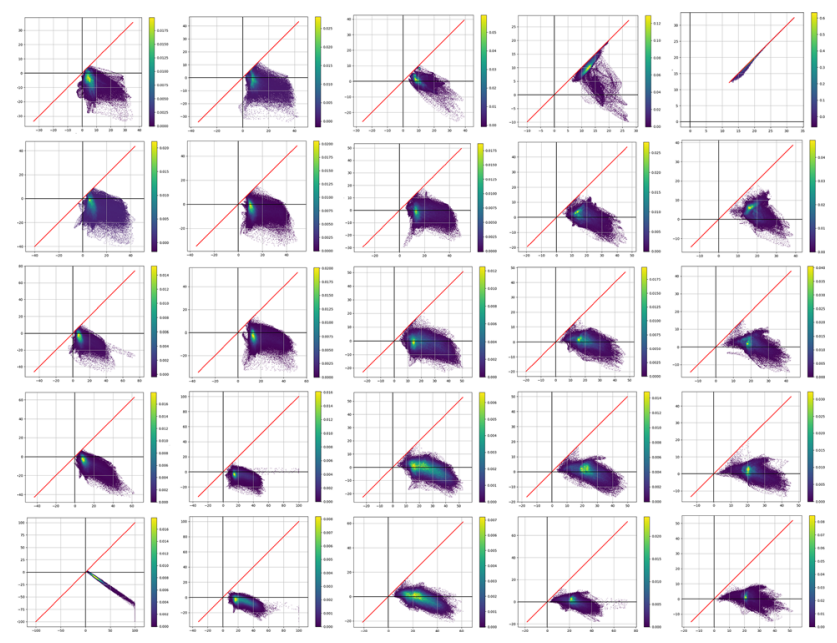

Fig. 9 Corresponding curvature diagrams (see Fig. 8). The bilinear interpolation of generation parameters is also reflected into the curvature diagrams as a continuous evolution. Please note that the curvature distributions of the four reference shapes concentrate around $(0,0)$ for layers, the identity half-line $\{y=x, x>0\}$ for spheres, the horizontal half-axis $\{y=0, x>0\}$ for tubes, and a half-line $\{y=-a x, x>0\}$ with $a>0$ for sponges. As explained in Sect. 4.1, this is expected from curvature diagrams

\section{discrepancy:}

$$
\mathrm{ND}=\frac{\left.\int_{\Omega}\left|\frac{\epsilon}{2}\right| \nabla u\right|^{2}-\frac{W(u)}{\epsilon} \mid \mathrm{d} x}{\int_{\Omega}\left(\frac{\epsilon}{2}|\nabla u|^{2}+\frac{W(u)}{\epsilon}\right) \mathrm{d} x} \in[0,1] .
$$

The discrepancy measures how much $u$ deviates from a phase-field with tanh profile as in (19), and is an indicator of good behavior in numerical experiments. In particular, it vanishes for functions with tanh profile. After normalization by the diffuse area, the normalized discrepancy varies between 0 and 1 , with 0 indicating a good quality in the numerical approximations. In Online Supplement, Figures 4 and 5, we compare the profiles of phase-fields $u$ with low or high normalized discrepancies to an ideal tanh profile (19).

Shapes were deemed viable if the normalized discrepancy was under the threshold 0.75 and if $\max (u)>0.1$ and $\min (u)<-0.1$ to ensure that the zero level set was defined. We chose a lenient threshold 0.75 in order to still include some interesting shape textures in this study, even if they departed from a tanh profile (see Online Supplement, Figure 5).

If the random value assigned to $\left(\mathbf{a}, m_{0}\right)$ produced a non-viable shape, a new value was drawn uniformly until obtaining a viable shape. We thus generated 1000 shapes meeting the criteria mentioned above. Following this procedure, 1792 random simulations were carried out in total, comprising 1000 viable ones and 792 non-viable ones (44\% of the total). In Figures 6, 7, and 8 of the Online Supplement, we show some preliminary results on the way high normalized discrepancies seem to be correlated with certain regions of parameters.

We computed the pairwise Wasserstein distance between their curvature diagrams, as described in the last paragraph of Sect. 4.1. To reduce the computation time, in each comparison we restricted the point cloud (25) to 10000 cells randomly taken from the whole mesh. The distance matrix was then given as input to UMAP [70], a manifold learning technique for dimension reduction, with the option metric $=$ 'precomputed'. The 1000 shapes were embedded in a 2D atlas by considering local neighborhoods of $n=25$ points, a minimal distance $m d=0.05$ between embedded points and a spread $s p=1$. For reproducibility, the random seed was set to $R S=1$. To enhance the visualization, the embedded points were labeled with Hdbscan [69], with a minimum cluster size $m c s=10$ and a minimum number of samples $m s=10$. They were colored according to their cluster number, and as black dots if Hdbscan classified them as noise. We picked some shapes from the point cloud and displayed their thumbnails, with their location specified by an arrow. Please note that the thumbnails do not exhaustively cover all the types of morphologies, but may give an idea of their disparity.

The atlas in Fig. 10 shows that the shape textures are roughly distributed into three main families, spheres (top, left), layers (top), and tubules (central part) that occupy the largest region. In two marginal regions, we also identified outliers, such as highly packed tubules (bottom, right), and fragmented shapes (bottom, left). This suggest that the selec- 


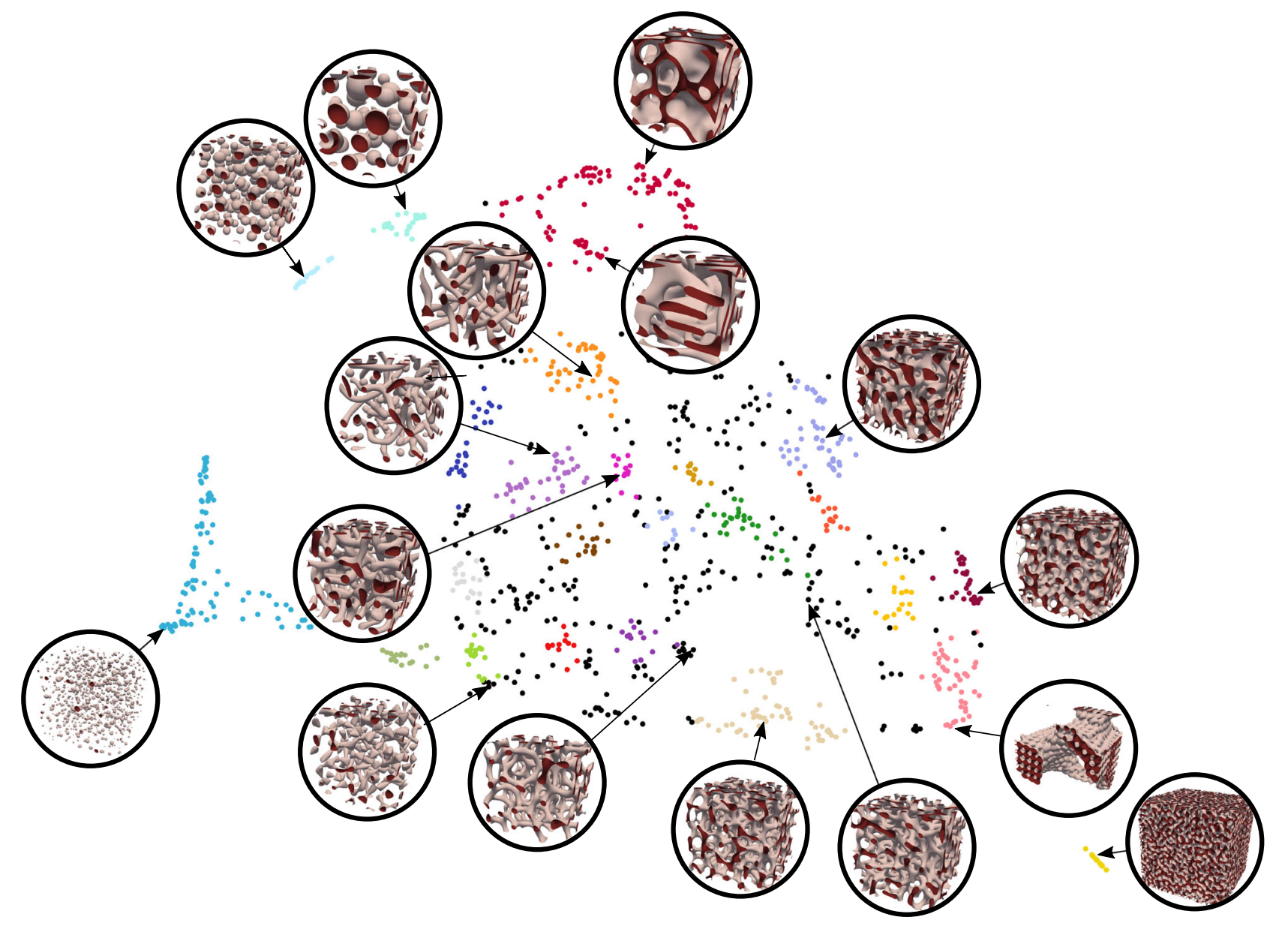

Fig. 10 Atlas of 1000 shapes visualized in UMAP. The generation parameters were chosen randomly and we applied some criteria to select mainly viable shapes. Three major families can be identified: spheres, layers, and tubules which constitute the principal type of shape textures. Let us highlight the large intra-variation in the family of tubules, that

tion criteria mentioned above were not selective enough for discarding badly-converged shapes (i.e. over-diffused phasefields), which is expected given the lenient threshold 0.75 . The marginal regions concentrate most of the outliers, but we also noticed a few of them spread inside the main regions. These shapes are characterized by high normalized discrepancies and irregular aspects (see Online Supplement, Figures 3 and 5).

The transition between morphological subtypes is quite smooth when moving continuously in the atlas; however, we did not examine in which way the generation parameters relate to the spatial embedding yet. The family of tubules has a large intra-variation, and features not only smooth sponges or long tubes, but also irregular, tortuous and anisotropic tubules that have a multi-scale texture, as mentioned in Experiments 2. This is a remarkable behavior of the gen- features not only smooth sponges and long tubes, but also irregular and anisotropic tubules with a multi-scale texture. This is a remarkable feature of the generation model, since all pixels of the simulation domain share identical properties with respect to the minimization problem

eration model, since in regard of the minimization problem, all points have the same homogeneous properties in space.

\section{Discussion}

In this final section, we discuss the strengths and limitations of the algorithm, propose a few extensions, and present the important implications of a unifying framework on applied contexts.

\subsection{Strengths and Limitations}

As seen in the simulations of Sect. 4, curvatubes leads to a wide range of membranous and tubular shapes, some of which have a multi-scale texture. The generation parameters and the curvature diagrams capture well the notion of 


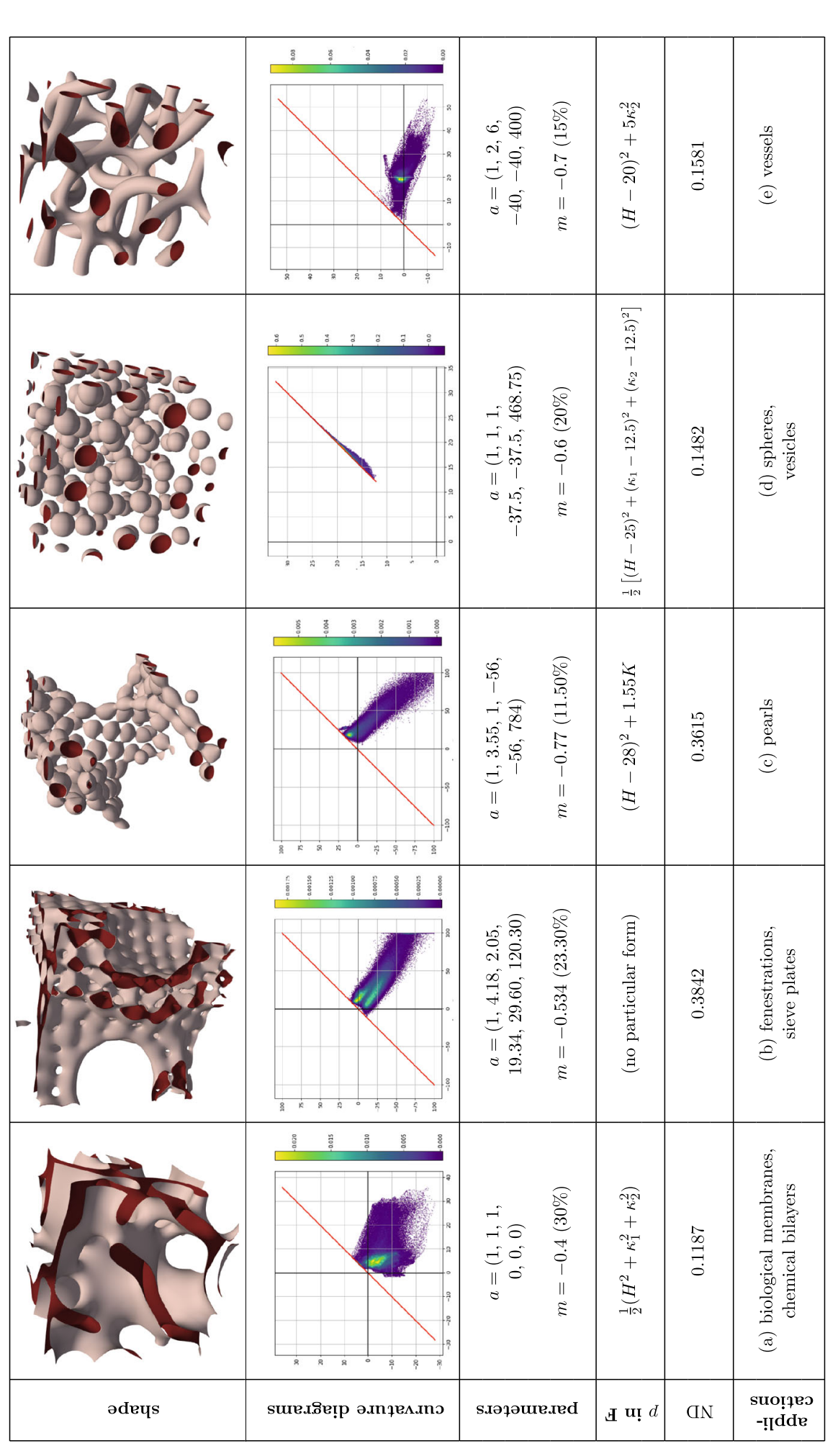

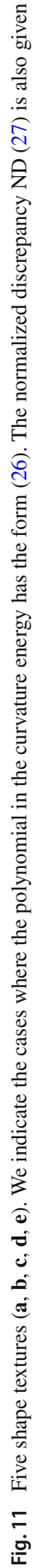




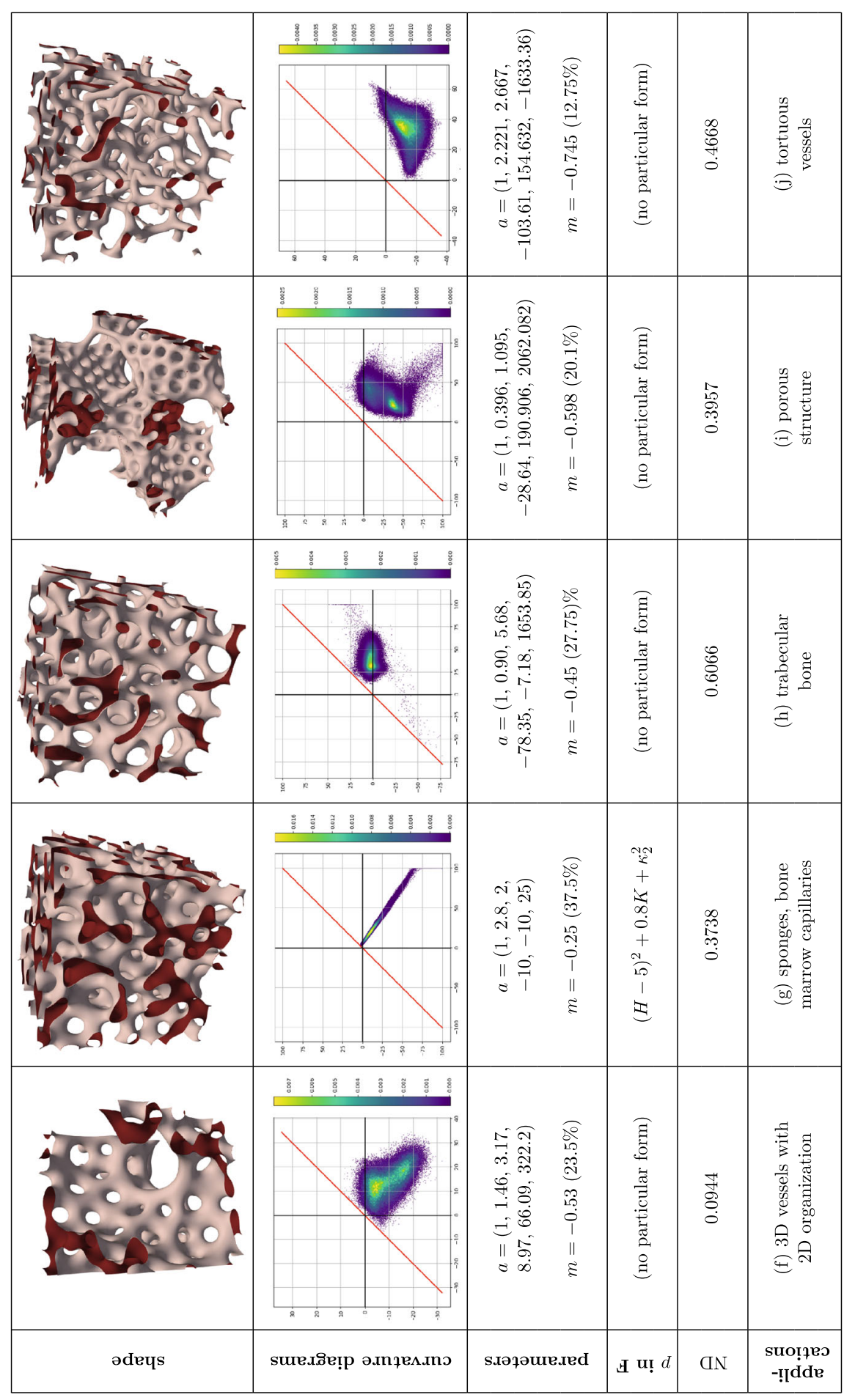

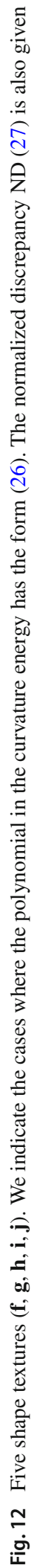


shape texture. The algorithm is GPU-accelerated and takes advantage of automatic differentiation combined to external algorithms (Adam, L-BFGS) to descend gradient flows. Contrarily to refined numerical schemes, its aim is not to precisely solve the evolution equation, but rather to converge fast to a local minimizer with small energy. The mathematical computation of the gradient is not required either. The coefficients can be chosen in a flexible way, without letting the algorithm diverge numerically, even in mathematically ill-posed cases. The simulation results are reproducible, and seem to behave in accordance with the polynomial of curvatures in the energy especially under the form (26); although much work is still needed to understand mathematically how different polynomial energies are linked to different shape textures, and what are the values of coefficients that correspond to well-posed geometric problems. We leave these difficult questions as future work.

Let us caution the reader that here, the model does not generate tree-like structures, for which junctions are hierarchically organized into parent and children nodes, and cycles are excluded. Thus, it cannot be applied to the respiratory system, and can only model vessels that branch and cycle a lot such as capillaries. This is because the model is devised primarily for reproducing shape texture, but not shape structure. Some extensions of the framework to include structured constraints are proposed in the next subsection. The notion of shape texture is inspired from visual texture in images, characterized by spatially repeated elements whose conformation, such as size, color, orientation, are subject to randomness $[46,62,85]$. Texture is hence a statistically defined property, while structure may be understood as an orthogonal component.

Furthermore, in contrast to the Helfrich biomembrane model and the FCH model of [41], which truly model some physico-chemical energy derived from microscopic interactions, we do not assume any such physical ground to the general curvature functional $\mathbf{F}$ and the corresponding phasefield functional $\mathscr{F}_{\epsilon}$ that we propose. This framework is simply intended to provide a descriptive tool to analyze tubular textures, and may be used to quantify biological shapes in terms of geometry, even without any knowledge of the underlying microscopic interactions.

\subsection{Extensions}

We can include a constraint on the orientation of the normal vector $\mathbf{n}_{\mathscr{S}}$ to the surface $\mathscr{S}$, by encouraging $\mathbf{n}_{\mathscr{S}}$ to be orthogonal to the direction associated to a vector $\theta$ of unit norm, as in

$$
\widetilde{\mathbf{F}}(\mathscr{S})=\int_{\mathscr{S}} p\left(\kappa_{1}, \kappa_{2}\right) \mathrm{d} A+\mu \int_{\mathscr{S}}\left(\theta \cdot \mathbf{n}_{\mathscr{S}}\right)^{2} \mathrm{~d} A .
$$

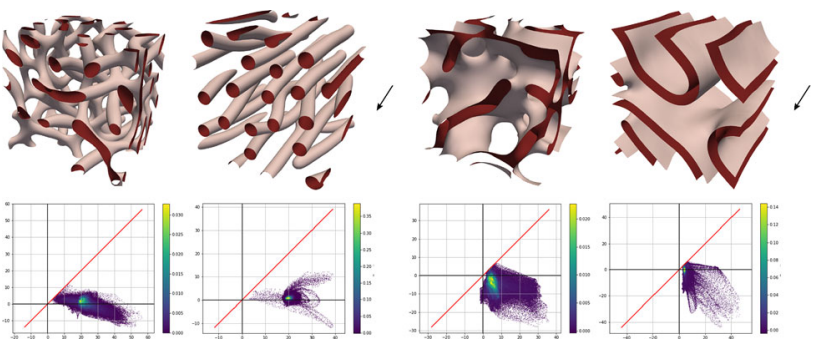

Fig. 13 Including orientation in the loss aligns tubes and layers. The tubes were generated with $\mathbf{a}=(1,2,6,-40,-40,400)$, which corresponds to $\mathbf{F}=(H-20)^{2}+5 \kappa_{2}^{2}, m_{0}=-0.6$, and $\mu=0$ or 1000 for the first and second shapes. The layers were generated using $\mathbf{a}=(1,1,1,0,0,0)$, which corresponds to $\mathbf{F}=\left(H^{2}+\kappa_{1}^{2}+\kappa_{2}^{2}\right) / 2$, $m_{0}=-0.4$, and $\mu=0$ or 800 for the third and fourth shapes. When $\mu \neq 0$, the direction of $\theta$ is indicated by the arrow. The curvature diagrams show that after alignment, the curvatures are more densely clustered around the dirac masses associated to perfectly cylindrical or perfectly flat shapes, namely, $\delta_{(20,0)}$ for tubes and $\delta_{(0,0)}$ for layers

This can be approximated by the phase-field energy

$$
\begin{aligned}
\widetilde{\mathscr{F}}_{\epsilon} & =\mathscr{F}_{\epsilon}+\mu \int_{\Omega}\left(\theta \cdot \mathbf{n}_{u}\right)^{2} \epsilon|\nabla u|^{2} \mathrm{~d} x \\
& =\mathscr{F}_{\epsilon}+\epsilon \mu \int_{\Omega}(\theta \cdot \nabla u)^{2} \mathrm{~d} x,
\end{aligned}
$$

where $\mathscr{F}_{\epsilon}$ is our phase-field energy constructed in Sect. 3.2. The effect on tubes is to align their median axis along $\theta$, while inciting flat layers to be parallel to $\theta$, as in Fig. 13.

Another way to give some structure to the shape is to use space-dependent generation parameters $(\mathbf{a}(x), m(x))$, i.e., make them spatialized instead of constant, as in Fig. 1. In the current version of the algorithm, since $u$ is periodic, the coefficients $\mathbf{a}(x)$ but also the mass $m$ are required to be periodic. The change of variable of Sect. 4.1 becomes

$u(x)=\nabla \cdot A(x)+m(x)$.

In Fig. 1, we took four reference generation parameters $p_{1}$, $p_{2}, p_{3}$, and $p_{4}$ (see Table 2), and linearly interpolated them along the horizontal axis, by taking into account the periodicity. We also repeated the first and last values (following the order $\left.p_{1}, p_{1}, p_{2}, p_{3}, p_{4}, p_{4}\right)$, and cropped the shape by dropping the first and last cubes. The spatialized parameters hence coincide with $p_{1}, p_{2}, p_{3}, p_{4}$ at the vertical midplanes of the four cubes delimited by the dashed lines.

Many other extensions to this framework are also possible and could be explored in the future. For instance, it is compatible with inclusion or exclusion constraints, by forcing $u$ to take positive or negative values in some spatial regions, so that the shape contains or excludes them. Another possibility is to extend the framework to phase-fields with three phases 
Table 2 Generation parameters used in the spatialized interpolation of Fig. 1, associated with the central regions of the four cubes delimited by dashed lines (from left to right)

\begin{tabular}{llll}
\hline $\mathbf{a}$ & $\mathbf{b}$ & $p$ in energy $\mathbf{F}$ & $m_{0}$ \\
\hline$(2,2,2,0,0,0)$ & $(1,0,0,1,0,1,0)$ & $H^{2}+\kappa_{1}^{2}+\kappa_{2}^{2}$ & $-0.4(30 \%)$ \\
$(1,2,6,-40,-40,400)$ & $(1,20,0,0,0,5,0)$ & $(H-20)^{2}+5 \kappa_{2}^{2}$ & $-0.6(20 \%)$ \\
$(2,2,2,-75,-75,937.5)$ & $(1,25,0,1,12.5,1,12.5)$ & $(H-25)^{2}+\left(\kappa_{1}-12.5\right)^{2}+\left(\kappa_{2}-12.5\right)^{2}$ & $-0.7(15 \%)$ \\
$(2,2,11,-180,-90,4050)$ & $(1,45,0,1,45,10,0)$ & $(H-45)^{2}+\left(\kappa_{1}-45\right)^{2}+10 \kappa_{2}^{2}$ & $-0.7(15 \%)$ \\
\hline
\end{tabular}

The vector $\mathbf{b}$ parameterizes the polyomial expression (26)

or more, to generate shapes intertwined in each other, without intersecting, while minimizing different curvature energies.

\subsection{Importance of a Unifying Theory, and Future Applications}

Finally, we have identified several implications that a generation model unifying tubular and membranous shapes could have in other contexts.

- Design bio-inspired shape textures and porous materials: the generation model could help in the design of bio-fabricated vascular networks for tissue regeneration [94]; the design of scaffolds for bone tissue engineering [36] and cellular solids in architecture [77], both inspired by the structure of bone trabeculae; or the design of porous materials $[59,111]$.

- Model morphological states and trajectories: if generation parameters can be inferred from morphological states, a morphological transformation can be modeled as a trajectory in the lower-dimensional space of parameters, and then analyzed as a longitudinal trajectory [31]. In particular, the biological transformations mentioned in the Introduction would be modeled in a continuous way.

- Provide regularization prior for tubular segmentation: the generation model could be included as a regularizing loss in variational segmentation methods of vascular structures [33,71,107], to select certain tubular morphologies against others. It could also be combined with $3 \mathrm{D}$ reconstruction from $2 \mathrm{D}$ slices methods $[13,53,64]$.

- Build a synthetic database of textures: the generation algorithm could provide, at a low cost, a complete panel of synthetic textures on which to test and train vascular shape analysis methods $[50,84,89]$, including topological analysis methods $[15,47,79]$, segmentation algorithms, microvascular blood flow simulations $[5,86]$, but also simulations in porous materials $[65,95,109]$. It could also provide a database to research in shape and texture perception [108]; the way we perceive shapes is intimately linked to the way we want to quantify them.
We of course did not cover all these applications here, but intend to focus on two of the points aforementioned as future work.

The first one is to model morphological states or trajectories of biological tissues as static values or trajectories of generation parameters $(\mathbf{a}(t), m(t))$, which supposes that parameters can be inferred from shapes. This can be done naively, by visual inspection and trial-and-error; or, by first producing an atlas of reference shapes densely sampling a region with the desired morphologies, similarly to Experiment 4 . Using the curvature diagram of the query shape $u_{0}$, the shapes closest to it in terms of the Wasserstein distance are found. We can then initialize $\left(\mathbf{a}, m_{0}\right)$ at these values, and minimize the loss

$\mathscr{F}_{\epsilon}\left[u_{0} ; \mathbf{a}, m_{0}\right]$

with respect to the parameters $\left(\mathbf{a}, m_{0}\right)$ instead of the phasefield $u_{0}$, by using nearly the same algorithmic framework as Algorithm 1.

The second related application is to include the curvature energy as a regularizing loss in order to segment vascular structures. The energy would then select certain tubular morphologies over others. This could be used for instance to reconstruct 3D tubular structures captured in several 2D images at different depths of a biological sample (as done in optical sectioning), provided that there are not filaments too thin compared to the diffusion width $\epsilon$. The method is most effective if there is some knowledge of the shape textures that need to be segmented, so that the parameters can be appropriately tuned by inference, as previously explained.

Supplementary Information The online version contains supplementary material available at https://doi.org/10.1007/s10851-021-010499.

Acknowledgements The author is thankful to Dominique Bonnet and Antoniana Batsivari for providing the images of vessels that inspired this work. She expresses her gratitude to Anthea Monod for her guidance, to Jean Feydy for helpful insights on the numerical aspects, and to Pierre Degond for his advice. Finally she acknowledges valuable discussions with Simon Masnou, Blanche Buet and Elie Bretin. This work was jointly funded by Imperial College London and The Francis Crick Institute through a $\mathrm{PhD}$ studentship. 


\section{Declarations}

Conflict of interest The author declares that she has no conflict of interest.

Open Access This article is licensed under a Creative Commons Attribution 4.0 International License, which permits use, sharing, adaptation, distribution and reproduction in any medium or format, as long as you give appropriate credit to the original author(s) and the source, provide a link to the Creative Commons licence, and indicate if changes were made. The images or other third party material in this article are included in the article's Creative Commons licence, unless indicated otherwise in a credit line to the material. If material is not included in the article's Creative Commons licence and your intended use is not permitted by statutory regulation or exceeds the permitted use, you will need to obtain permission directly from the copyright holder. To view a copy of this licence, visit http://creativecomm ons.org/licenses/by/4.0/.

\section{Appendix: Proof of the $\Gamma$-Limsup}

The proof of Theorem 2 consists in showing that the sequence constructed in $[8,9]$ satisfies the theorem for our extended formulation $\mathscr{F}_{\epsilon}$.

Let us first recall their construction (up to a factor $\sqrt{2}$ ). By assumption, the surface $\mathscr{S}=\partial E \cap \Omega$ is $C^{2}$. Let $d: \Omega \rightarrow \mathbb{R}$ be the signed distance function to $\mathscr{S}$, with the convention that $d$ is positive inside $E$ and negative on $\Omega \backslash \bar{E}$. Let $\gamma_{\epsilon}$ be defined on $\mathbb{R}$ by

$\gamma_{\epsilon}(s)=\left\{\begin{array}{cl}\tanh \left(\frac{s}{\sqrt{2} \epsilon}\right) & \text { if } s \in\left[0, b_{\epsilon}\right) \\ p_{\epsilon}(s) & \text { if } s \in\left[b_{\epsilon}, c_{\epsilon}\right) \\ +1 & \text { if } s \in\left[c_{\epsilon},+\infty\right) \\ -\gamma_{\epsilon}(-s) & \text { if } \quad s<0\end{array}\right.$,

where $b_{\epsilon}=\sqrt{2} \epsilon|\log \epsilon|$ and $c_{\epsilon}=\sqrt{2}\left(\epsilon+\epsilon^{3}+\epsilon|\log \epsilon|\right)$, $a_{\epsilon}=\frac{1}{\left(1+\epsilon^{2}\right)^{3}}$, and $p_{\epsilon}(s)=1-a_{\epsilon}\left(s-c_{\epsilon}\right)^{2}$ is a parabolic arc connecting the graphs of $\tanh \left(\frac{s}{\sqrt{2} \epsilon}\right)$ and the constant +1 on the interval $\left(b_{\epsilon}, c_{\epsilon}\right)$. The coefficients $a_{\epsilon}$ and $c_{\epsilon}$ ensure that $\gamma_{\epsilon} \in H^{2}(\mathbb{R})$. Now, we set

$u_{\epsilon}=\gamma_{\epsilon} \circ d \in W^{2,2}(\Omega)$.

We split $\Omega$ into three regions, $\Omega_{\epsilon}^{(1)}=\left\{0<|d|<b_{\epsilon}\right\}$, $\Omega_{\epsilon}^{(2)}=\left\{b_{\epsilon}<|d|<c_{\epsilon}\right\}$ and $\Omega_{\epsilon}^{(3)}=\left\{c_{\epsilon}<|d|\right\}$ (on which $u_{\epsilon}= \pm 1$ ). On $\Omega_{\epsilon}^{(1)}$ and $\Omega_{\epsilon}^{(2)}, \nabla u_{\epsilon} \neq 0$ and $\mathscr{M}_{u_{\epsilon}}^{\epsilon}=\epsilon\left|u_{\epsilon}\right| B_{u_{\epsilon}}^{\epsilon}$, whereas on $\Omega_{\epsilon}^{(3)}, \nabla u_{\epsilon}=0$ and $\mathscr{M}_{u_{\epsilon}}^{\epsilon}=0$. Note that the region $\overline{\Omega_{\epsilon}^{(1)} \cup \Omega_{\epsilon}^{(2)}}=\left\{d \leq c_{\epsilon}\right\}$ decreases and concentrates around the surface $\mathscr{S}$ as $\epsilon \rightarrow 0$, while the complementary region $\Omega_{\epsilon}^{(3)}$ grows. Also, on $\Omega_{\epsilon}^{(2)},\left|\nabla u_{\epsilon}\right|$ is bounded by $p_{\epsilon}^{\prime}\left(b_{\epsilon}\right)=\frac{2 \sqrt{2} \epsilon}{\left(1+\epsilon^{2}\right)^{2}}$.

As $\mathscr{S}$ is compact and $C^{2}$, there exists a tubular neigh$\operatorname{borhood} \operatorname{Tub}\left(\mathscr{S}, d_{0}\right)=\left\{d \leq d_{0}\right\}$ of $\mathscr{S}$ on which $d$ is
$\mathscr{C}^{2}$ [58], and for any point $z$ in $\operatorname{Tub}\left(\mathscr{S}, d_{0}\right)$ the distance $d(z)$ is realized by a unique point $\pi_{\mathscr{S}}(z)$ which satisfies $z=\pi_{\mathscr{S}}(z)+d(z) N\left(\pi_{\mathscr{S}}(z)\right)$. On the tubular neighborhood, the signed distance satisfies the eikonal equation $|\nabla d|=1$, implying that Hess $d \nabla d=0$. The symmetric matrix -Hess $d(x)^{8}$ has two eigenvalues $\lambda_{1}(x) \geq \lambda_{2}(x)$ corresponding to the principal directions of the associated level set, and a third eigenvalue 0 in the direction $\pm \nabla d$. The eigenvalues $\lambda_{1}$ and $\lambda_{2}$ are continuous on $\operatorname{Tub}\left(\mathscr{S}, d_{0}\right)$.

To show (21), as $\left|u_{\epsilon}-\left(2 \chi_{E}-1\right)\right| \leq 1$,

$$
\begin{aligned}
\int_{\Omega}\left|u_{\epsilon}-\left(2 \chi_{E}-1\right)\right| \mathrm{d} x & =\int_{\left\{|d|<c_{\epsilon}\right\}}\left|u_{\epsilon}-\left(2 \chi_{E}-1\right)\right| \mathrm{d} x \\
& \leq \mathscr{L}^{3}\left(\left\{|d|<c_{\epsilon}\right\}\right) \rightarrow 0
\end{aligned}
$$

so $\lim u_{\epsilon}=2 \chi_{E}-1$ in $L^{1}(\Omega)$ and it remains to prove (22) and (23).

Let $\phi \in C_{c}^{0}(\Omega)$ be a continuous function with compact support. We need to show that $\int_{\Omega} \epsilon\left|\nabla u_{\epsilon}\right|^{2} \phi \mathrm{d} x$ converges to $\sigma \int_{\mathscr{S}} \phi d \mathscr{H}^{2}$ as $\epsilon$ is sent to zero. First, we work only on $\Omega_{\epsilon}^{(1)}$, as

$$
\begin{aligned}
\int_{\Omega} \epsilon\left|\nabla u_{\epsilon}\right|^{2} \phi \mathrm{d} x= & \int_{\Omega_{\epsilon}^{(1)}} \epsilon\left|\nabla u_{\epsilon}\right|^{2} \phi \mathrm{d} x \\
& +\int_{\Omega_{\epsilon}^{(2)}} \epsilon\left|\nabla u_{\epsilon}\right|^{2} \phi \mathrm{d} x
\end{aligned}
$$

and the second integral tends to zero since the integrand is bounded and $\mathscr{L}^{3}\left(\Omega_{\epsilon}^{(2)}\right) \rightarrow 0$. By the co-area formula,

$$
\begin{aligned}
& \int_{\Omega_{\epsilon}^{(1)}} \epsilon\left|\nabla u_{\epsilon}\right|^{2} \phi \mathrm{d} x \\
& =\int_{-1}^{u_{\epsilon}\left(b_{\epsilon}\right)}\left(\int_{\left\{u_{\epsilon}=t\right\}} \epsilon\left|\nabla u_{\epsilon}\right| \phi d \mathscr{H}^{2}\right) \mathrm{d} t \\
& =\int_{-1}^{u_{\epsilon}\left(b_{\epsilon}\right)} \sqrt{2 W(t)}\left(\int_{\left\{u_{\epsilon}=t\right\}} \phi d \mathscr{H}^{2}\right) \mathrm{d} t,
\end{aligned}
$$

where we use $\left|\nabla u_{\epsilon}\right|=\frac{\left(1-u_{\epsilon}^{2}\right)}{\sqrt{2} \epsilon}=\frac{\sqrt{2 W(t)}}{\epsilon}$ on $\Omega_{\epsilon}^{(1)}$. Therefore,

$$
\begin{aligned}
& \left.\left|\int_{\Omega_{\epsilon}^{(1)}} \epsilon\right| \nabla u_{\epsilon}\right|^{2} \phi \mathrm{d} x-\sigma \int_{\mathscr{S}} \phi d \mathscr{H}^{2} \mid \\
& \leq \int_{-1}^{u_{\epsilon}\left(b_{\epsilon}\right)} \sqrt{2 W(t)} \mid \int_{\left\{d=\gamma_{\epsilon}^{-1}(t)\right\}} \phi d \mathscr{H}^{2} \\
& \quad-\int_{\{d=0\}} \phi d \mathscr{H}^{2} \mid \mathrm{d} t
\end{aligned}
$$

8 The minus sign correspond to the convention that if $\partial E$ is a sphere, the eigenvalues should be positive. 


$$
+\int_{\mathscr{S}} \phi d \mathscr{H}^{2} \int_{u_{\epsilon}\left(b_{\epsilon}\right)}^{1} \sqrt{2 W(t)} \mathrm{d} t
$$

where $\gamma_{\epsilon}^{-1}:(-1,1) \rightarrow\left(-c_{\epsilon}, c_{\epsilon}\right)$ denotes the inverse of the restriction of $\gamma_{\epsilon}$ to $\left(-c_{\epsilon}, c_{\epsilon}\right)$. Using $u_{\epsilon}\left(b_{\epsilon}\right)=\frac{1-\epsilon^{2}}{1+\epsilon^{2}} \rightarrow 1$, the last term goes to zero.

The convergence to zero of the bound is proved if for any $\delta>0$, we can find $\epsilon_{0}$ small enough such that $\forall \epsilon<\epsilon_{0}$, $\forall s \in\left(-c_{\epsilon}, c_{\epsilon}\right)$,

$$
\left|\int_{\{d=s\}} \phi d \mathscr{H}^{2}-\int_{\mathscr{S}} \phi d \mathscr{H}^{2}\right| \leq \delta .
$$

This is true because, if $p \in \mathscr{S}$, there is a $C^{1}$-diffeomorphism $\psi$ mapping $U \times\left(-d_{0}, d_{0}\right)$ onto $\operatorname{Tub}\left(V, d_{0}\right)$, where $U \subset \mathbb{R}^{2}$ is open and $V \subset \mathscr{S}$ is an open neighborhood of $p$. For $s \in\left(-d_{0}, d_{0}\right)$, by the change of variables formula,

$$
\begin{aligned}
& \int_{\{d=s\} \cap \operatorname{Tub}\left(V, d_{0}\right)} \phi d \mathscr{H}^{2} \\
& =\int_{U} \phi(\psi(x, y, s)) \sqrt{\left|\operatorname{det}\left(\operatorname{Jac}_{\psi}^{T} \operatorname{Jac}_{\psi}\right)\right|}(x, y, s) \mathrm{d} x \mathrm{~d} y
\end{aligned}
$$

where $\operatorname{Jac}(\psi)=\left(\frac{\partial \psi}{\partial x}, \frac{\partial \psi}{\partial y}\right) \in \mathbb{R}^{3 \times 2}$, and this integral converges to $\int_{V} \phi d \mathscr{H}^{2}$ when $s \rightarrow 0$. By compactness, we can consider a finite number of such neighborhoods $V$ and conclude that the limit (22) holds.

Now, we prove the convergence of the energies. It can be shown that on $\Omega_{\epsilon}^{(1)}$, we have

$$
\begin{aligned}
\mathscr{B}_{u_{\epsilon}}^{\epsilon} & =- \text { Hess } d \\
\left\|\mathscr{B}_{u_{\epsilon}}^{\epsilon}\right\|^{2} & =\lambda_{1}^{2}+\lambda_{2}^{2} \\
\operatorname{Tr} \mathscr{B}_{u_{\epsilon}}^{\epsilon} & =-\Delta d=\lambda_{1}+\lambda_{2}
\end{aligned}
$$

and on $\Omega_{\epsilon}^{(2)}$

$$
\begin{aligned}
\mathscr{B}_{u_{\epsilon}}^{\epsilon} & =-\operatorname{Hess} d+f_{\epsilon} \mathbf{n}_{d} \otimes \mathbf{n}_{d} \\
\left\|\mathscr{B}_{u_{\epsilon}}^{\epsilon}\right\|^{2} & =\lambda_{1}^{2}+\lambda_{2}^{2}+f_{\epsilon}^{2} \\
\operatorname{Tr} \mathscr{B}_{u_{\epsilon}}^{\epsilon} & =-\Delta d+f_{\epsilon}=\lambda_{1}+\lambda_{2}+f_{\epsilon}
\end{aligned}
$$

where we define the auxiliary function

$$
f_{\epsilon}=\frac{1}{p_{\epsilon}^{\prime}(d)}\left(p_{\epsilon}^{\prime \prime}(d)-\frac{W^{\prime}\left(p_{\epsilon}(d)\right)}{\epsilon^{2}}\right) .
$$

such that $f_{\epsilon}\left|\nabla u_{\epsilon}\right| \leq \frac{2}{\left(1+\epsilon^{2}\right)^{3}}+\frac{4}{1+\epsilon^{2}}$ remains bounded on $\Omega_{\epsilon}^{(2)}$.

It is sufficient to show that the limit holds independently for each term of $F$. We prove it for the term $\kappa_{1}^{2}$ associated to $\mathbf{a}=(1,0,0,0,0,0)$. The phase-field functional writes

$$
\begin{aligned}
\mathscr{F}_{\epsilon}= & \frac{1}{2 \epsilon} \int_{\Omega}\left(\left\|\mathscr{M}_{u_{\epsilon}}^{\epsilon}\right\|^{2}\right. \\
& \left.+\operatorname{Tr} \mathscr{M}_{u_{\epsilon}}^{\epsilon} \sqrt{\left(2\left\|\mathscr{M}_{u}^{\epsilon}\right\|^{2}-\left(\operatorname{Tr} \mathscr{M}_{u}^{\epsilon}\right)^{2}\right)^{+}}\right) \mathrm{d} x .
\end{aligned}
$$

Using the relationships between $\mathscr{M}_{u}^{\epsilon}, \mathscr{B}_{u_{\epsilon}}^{\epsilon}$ and Hess $d$, on $\Omega_{\epsilon}^{(1)}$ we have $2\left\|\mathscr{M}_{u}^{\epsilon}\right\|^{2}-\left(\operatorname{Tr} \mathscr{M}_{u}^{\epsilon}\right)^{2}=\left(\lambda_{1}-\lambda_{2}\right)^{2} \geq 0$ and

$$
\begin{aligned}
& \left\|\mathscr{M}_{u_{\epsilon}}^{\epsilon}\right\|^{2}+\operatorname{Tr} \mathscr{M}_{u_{\epsilon}}^{\epsilon} \sqrt{\left(2\left\|\mathscr{M}_{u}^{\epsilon}\right\|^{2}-\left(\operatorname{Tr} \mathscr{M}_{u}^{\epsilon}\right)^{2}\right)^{+}} \\
& =2 \epsilon^{2}\left|\nabla u_{\epsilon}\right|^{2} \lambda_{1}^{2},
\end{aligned}
$$

while on $\Omega_{\epsilon}^{(2)}$, we have $\xi:=2\left\|\mathscr{M}_{u}^{\epsilon}\right\|^{2}-\left(\operatorname{Tr} \mathscr{M}_{u}^{\epsilon}\right)^{2}=\lambda_{1}^{2}+$ $\lambda_{2}^{2}+f_{\epsilon}^{2}-2 \lambda_{1} \lambda_{2}-2 \lambda_{1} f_{\epsilon}-2 \lambda_{2} f_{\epsilon}$. As $\xi^{+} \leq|\xi| \leq 3\left(\lambda_{1}^{2}+\right.$ $\left.\lambda_{2}^{2}+f_{\epsilon}^{2}\right) \leq 3\left(\left|\lambda_{1}\right|+\left|\lambda_{2}\right|+\left|f_{\epsilon}\right|\right)^{2}$, we get the bound

$$
\begin{aligned}
& \left|\operatorname{Tr} \mathscr{M}_{u_{\epsilon}}^{\epsilon} \sqrt{\left(2\left\|\mathscr{M}_{u}^{\epsilon}\right\|^{2}-\left(\operatorname{Tr} \mathscr{M}_{u}^{\epsilon}\right)^{2}\right)^{+}}\right| \\
& \leq \sqrt{3}\left(\left|\lambda_{1}\right|+\left|\lambda_{2}\right|+\left|f_{\epsilon}\right|\right)^{2}
\end{aligned}
$$

$\mathscr{F}_{\epsilon}$ can thus be decomposed into $\mathscr{F}_{\epsilon}=I_{\epsilon}+J_{\epsilon}$, where

$$
\begin{aligned}
I_{\epsilon}= & \int_{\Omega_{\epsilon}^{(1)} \cup \Omega_{\epsilon}^{(2)}} \epsilon\left|\nabla u_{\epsilon}\right|^{2} \lambda_{1}^{2}(x) \mathrm{d} x, \quad \text { and } \\
\left|J_{\epsilon}\right| \leq & \int_{\Omega_{\epsilon}^{(2)}} \epsilon\left|\nabla u_{\epsilon}\right|^{2} \\
& \times\left(\lambda_{2}^{2}+f_{\epsilon}^{2}+\sqrt{3}\left(\left|\lambda_{1}\right|+\left|\lambda_{2}\right|+\left|f_{\epsilon}\right|\right)^{2}\right) \mathrm{d} x .
\end{aligned}
$$

On $\Omega_{\epsilon}^{(2)}$, the functions $\left|\nabla u_{\epsilon}\right|, f_{\epsilon}\left|\nabla u_{\epsilon}\right|,\left|\lambda_{1}\right|$ and $\left|\lambda_{2}\right|$ are all bounded. Therefore, as $\mathscr{L}^{3}\left(\Omega_{\epsilon}^{(2)}\right) \rightarrow 0$, we get $J_{\epsilon} \rightarrow 0$.

Finally, since $\left|\nabla u_{\epsilon}\right|=0$ on $\Omega_{\epsilon}^{(3)}$,

$I_{\epsilon}=\int_{\Omega} \epsilon\left|\nabla u_{\epsilon}\right|^{2}{\widetilde{\lambda_{1}}}^{2} \mathrm{~d} x$

where $\tilde{\lambda_{1}} \in \mathscr{C}_{c}^{0}(\Omega)$ continuously extends $\lambda_{1}$ beyond $\operatorname{Tub}\left(\mathscr{S}, d_{0}\right)$. Due to the convergence of Radon measures (22), this integral converges towards

$\lim _{\epsilon \rightarrow 0} I_{\epsilon}=\sigma \int_{\mathscr{S}} \kappa_{1}^{2} d \mathscr{H}^{2}$.

We can similarly prove the convergence for the other monomials, and therefore conclude that Theorem 2 holds.

\section{References}

1. Acquaah, F., Robson Brown, K.A., Ahmed, F., Jeffery, N., Abel, R.L.: Early trabecular development in human vertebrae: overproduction, constructive regression, and refinement. 
Front. Endocrinol. 6,(2015). https://doi.org/10.3389/fendo.2015. 00067. https://www.frontiersin.org/articles/10.3389/fendo.2015. 00067/full. Publisher: Frontiers

2. Aggarwal, C.C., Hinneburg, A., Keim, D.A.: On the surprising behavior of distance metrics in high dimensional space. In: J. Van den Bussche, V. Vianu (eds.) Database Theory: ICDT 2001, Lecture Notes in Computer Science, pp. 420-434. Springer, Berlin, Heidelberg (2001). https://doi.org/10.1007/3-540-44503X_27

3. Alberti, G.: Variational models for phase transitions, an approach via Gamma-convergence. In: Buttazzo, G., Marino, A., Murthy, M.K.V. (eds.) Calculus of Variations and Partial Differential Equations, pp. 95-114. Springer, Berlin Heidelberg (2000). https://doi. org/10.1007/978-3-642-57186-2_3

4. Ambrosio, L., Fusco, N., Pallara, D.: Functions of Bounded Variation and Free Discontinuity Problems. Oxford Mathematical Monographs. Oxford University Press, Oxford, New York (2000)

5. Balogh, P., Bagchi, P.: Direct numerical simulation of cellularscale blood flow in 3D microvascular networks. Biophys. J. 113(12), 2815-2826 (2017). https://doi.org/10.1016/j.bpj. 2017.10.020. https://www.sciencedirect.com/science/article/pii/ S0006349517311359

6. Bauer, M., Kuwert, E.: Existence of minimizing Willmore surfaces of prescribed genus. Int. Math. Res. Not. 2003(10), 553-576 (2003). https://doi.org/10.1155/S1073792803208072

7. Bellettini, G., Mugnai, L.: On the approximation of the elastica functional in radial symmetry. Calc. Var. Partial. Differ. Equ. 24(1), 1-20 (2005). https://doi.org/10.1007/s00526-004-0312-7

8. Bellettini, G., Mugnai, L.: Approximation of helfrichs functional via diffuse interfaces. SIAM J. Math. Anal. 42(6), 2402-2433 (2010). https://doi.org/10.1137/09077549X

9. Bellettini, G., Paolini, M.: Approssimazione variazionale di funzionali con curvatura, pp. 87-97. Seminario Analisi Matematica Univ. Bologna, Tecnoprint pp (1993)

10. Bertalmio, M., Sapiro, G., Caselles, V., Ballester, C.: Image inpainting. In: Computer Graphics, p. 8 (2000)

11. Beyer, K., Goldstein, J., Ramakrishnan, R., Shaft, U.: When Is "Nearest. In: Neighbor" Meaningful? In: C. Beeri, P. Buneman, (ed.) Database Theory: ICDT'99. Lecture Notes in Computer Science, pp. 217-235. Springer, Berlin, Heidelberg (1999). https:// doi.org/10.1007/3-540-49257-7_15

12. Braides, A.: Gamma-convergence for beginners. Oxford lecture series in mathematics and its applications. Oxford University Press, New York (2002)

13. Bretin, E., Dayrens, F., Masnou, S.: Volume reconstruction from slices. SIAM J. Imag. Sci. 10, 2326-2358 (2017)

14. Bretin, E., Masnou, S., Oudet, E.: Phase-field approximations of the Willmore functional and flow. Numer. Math. 131(1), 115-171 (2015). https://doi.org/10.1007/s00211-014-0683-4

15. Byrne, H.M., Harrington, H.A., Muschel, R., Reinert, G., Stolz, B.J., Tillmann, U.: Topological methods for characterising spatial networks: a case study in tumour vasculature (2019). arXiv: 1907.08711

16. Cahn, J.W., Hilliard, J.E.: Free energy of a nonuniform system. I. Interfacial free energy. J. Chem. Phys. 28(2), 258-267 (1958). https://doi.org/10.1063/1.1744102

17. Campelo, F., Arnarez, C., Marrink, S.J., Kozlov, M.M.: Helfrich model of membrane bending: from Gibbs theory of liquid interfaces to membranes as thick anisotropic elastic layers. Adv. Colloid Interf. Sci. 208, 25-33 (2014). https://doi.org/10. 1016/j.cis.2014.01.018. https://linkinghub.elsevier.com/retrieve/ pii/S000186861400030X

18. Canham, P.B.: The minimum energy of bending as a possible explanation of the biconcave shape of the human red blood cell. J. Theor. Biol. 26(1), 61-81 (1970). https://doi.org/10.1016/S0022- 5193(70)80032-7.http://www.sciencedirect.com/science/article/ pii/S0022519370800327

19. Chicco-Ruiz, A., Morin, P., Pauletti, M.S.: The shape derivative of the Gauss curvature. Revista de la Unión Matemática Argentina pp. 311-337 (2018). https://doi.org/10.33044/revuma.v59n2a06. http://inmabb.criba.edu.ar/revuma/revuma.php?p=doi/v59n2a06

20. Christlieb, A., Jones, J., Promislow, K., Wetton, B., Willoughby, M.: High accuracy solutions to energy gradient flows from material science models. J. Comput. Phys. 257, 193215 (2014). https://doi.org/10.1016/j.jcp.2013.09.049. https:// linkinghub.elsevier.com/retrieve/pii/S0021999113006633

21. Christlieb, A.J., Kraitzman, N., Promislow, K.: Competition and complexity in amphiphilic polymer morphology. Phys. D 400 (2019)

22. Cowan, C.: The Cahn-Hilliard equation as a gradient flow. $\mathrm{PhD}$ Thesis, Simon Fraser University (2005)

23. Dai, S., Promislow, K.: Geometric evolution of bilayers under the functionalized Cahn-Hilliard equation. Proc. R. Soc. A Math. Phys. Eng. Sci. 469(2153), 20120505 (2013). https://doi.org/10. 1098/rspa.2012.0505

24. De Giorgi, E.: Some remarks on Gamma-convergence and least squares method. In: Dal Maso, G., Dell'Antonio, G.F. (eds.) Composite Media and Homogenization Theory, pp. 135-142. Birkhäuser Boston (1991). https://doi.org/10.1007/978-1-46846787-1_8

25. Deuling, H.J., Helfrich, W.: Red blood cell shapes as explained on the basis of curvature elasticity. Biophys. J. 16(8), 861-8 (1976)

26. Doğan, G., Nochetto, R.H.: First variation of the general curvature-dependent surface energy. ESAIM Math. Model. Numer. Anal. 46(1), 59-79 (2012). https://doi.org/10.1051/m2an/ 2011019

27. Du, Q., Liu, C., Ryham, R., Wang, X.: Diffuse interface energies capturing the euler number: relaxation and renomalization. Commun. Math. Sci. 5(1), 233-242 (2007). https://projecteuclid. org:443/euclid.cms/1175797629. Publisher: International Press of Boston

28. Du, Q., Liu, C., Wang, X.: A phase field approach in the numerical study of the elastic bending energy for vesicle membranes. J. Comput. Phys. 198(2), 450-468 (2004). https://doi.org/10.1016/ j.jcp.2004.01.029. http://www.sciencedirect.com/science/article/ pii/S0021999104000373

29. Du, Q., Liu, C., Wang, X.: Retrieving topological information for phase field models. SIAM J. Appl. Math. 65(6), 1913-1932 (2005). https://doi.org/10.1137/040606417

30. Duarte, D., Hawkins, E.D., Akinduro, O., Ang, H., De Filippo, K., Kong, I.Y., Haltalli, M., Ruivo, N., Straszkowski, L., Vervoort, S.J., McLean, C., Weber, T.S., Khorshed, R., Pirillo, C., Wei, A., Ramasamy, S.K., Kusumbe, A.P., Duffy, K., Adams, R.H., Purton, L.E., Carlin, L.M., Lo Celso, C.: Inhibition of endosteal vascular niche remodeling rescues hematopoietic stem cell loss in AML. Cell Stem Cell 22(1), 64-77.e6 (2018). https://doi.org/10.1016/ j.stem.2017.11.006. https://linkinghub.elsevier.com/retrieve/pii/ S1934590917304587

31. Durrleman, S., Pennec, X., Trouvé, A., Braga, J., Gerig, G., Ayache, N.: Toward a comprehensive framework for the spatiotemporal statistical analysis of longitudinal shape data. Int. J. Comput. Vis. 103(1), 22-59 (2013). https://doi.org/ 10.1007/s11263-012-0592-x. http://link.springer.com/10.1007/ s11263-012-0592-x

32. Döbereiner, H.G., Selchow, O., Lipowsky, R.: Spontaneous curvature of fluid vesicles induced by trans-bilayer sugar asymmetry. Eur. Biophys. J. 28, 174-178 (1999). https://doi.org/10.1007/ s002490050197

33. El-Zehiry, N.Y., Grady, L.: In: Vessel segmentation using 3D elastica regularization, pp. 1288-1291. IEEE, Barcelona, Spain (2012). https://doi.org/10.1109/ISBI.2012.6235798 
34. Elliott, C.M.: The Cahn-Hilliard Model for the Kinetics of Phase Separation. In: Rodrigues, J.F. (ed.) Mathematical models for phase change problems, pp. 35-73. , Birkhäuser Basel, Basel (1989). https://doi.org/10.1007/978-3-0348-9148-6_3

35. Evans, L.C.: Partial differential equations, 2nd edn. No. v. 19 in Graduate studies in mathematics. American Mathematical Society, Providence, R.I (2010). OCLC: ocn465190110

36. Fantini, M., Curto, M., De Crescenzio, F.: A method to design biomimetic scaffolds for bone tissue engineering based on Voronoi lattices. Virt. Phys. Prototyp. 11(2), 77-90 (2016). https:// doi.org/10.1080/17452759.2016.1172301

37. Feydy, J.: Geometric data analysis, beyond convolutions, $\mathrm{PhD}$ Thesis. Université Paris-Saclay (2020)

38. Feydy, J., Séjourné, T., Vialard, F.X., Amari, S.i., Trouve, A., Peyré, G.: Interpolating between Optimal Transport and MMD using Sinkhorn Divergences. Presented at the 22nd International Conference on Artificial Intelligence and Statistics, pp. 26812690 (2019)

39. Fletcher, R.: Practical methods of optimization, 2nd edn. Wiley, Chichester; New York (1987)

40. Frost, M., Rahbek, E., Ejersted, C., Høilund-Carlsen, P., Bygum, A., Thomsen, J., Andreasen, C., Andersen, T., Frederiksen, A.: Modeling-based bone formation transforms trabeculae to cortical bone in the sclerotic areas in BuschkeOllendorff syndrome. A case study of two females with LEMD3 variants. Bone 135, 115313 (2020). https://doi.org/10.1016/ j.bone.2020.115313. http://linkinghub.elsevier.com/retrieve/pii/ S8756328220300934

41. Gavish, N., Jones, J., Xu, Z., Christlieb, A.J., Promislow, K.: Variational models of network formation and ion transport: applications to perfluorosulfonate ionomer membranes. Polymers 4, 630-655 (2012)

42. Goldman, R.: Curvature formulas for implicit curves and surfaces. Comput. Aided Geomet. Des. 22(7), 632-658 (2005). https://doi. org/10.1016/j.cagd.2005.06.005. http://www.sciencedirect.com/ science/article/pii/S0167839605000737

43. Gruber, A., Toda, M., Tran, H.: On the variation of curvature functionals in a space form with application to a generalized Willmore energy. Ann. Glob. Anal. Geom. 56(1), 147-165 (2019). https:// doi.org/10.1007/s10455-019-09661-0

44. Helfrich, W.: Elastic properties of lipid bilayers: theory and possible experiments. Zeitschrift fur Naturforschung Teil C Biochem. Biophys. Biol. Virol. 28(11), 693-703 (1973). https://doi.org/10. 1515/znc-1973-11-1209

45. Hsu, L., Kusner, R., Sullivan, J.: Minimizing the squared mean curvature integral for surfaces in space forms. Exp. Math. 1(3), 191-207 (1992). https://projecteuclid.org:443/euclid.em/ 1048622023. Publisher: A K Peters, Ltd

46. Julesz, B.: Visual pattern discrimination. IEEE Trans. Inf. Theory 8(2), 84-92 (1962). https://doi.org/10.1109/TIT.1962.1057698

47. Kanari, L., Dłotko, P., Scolamiero, M., Levi, R., Shillcock, J., Hess, K., Markram, H.: A topological representation of branching neuronal morphologies. Neuroinformatics (2018). https://doi.org/ 10.1007/s12021-017-9341-1

48. Kazhdan, M., Bolitho, M., Hoppe, H.: Poisson surface reconstruction. In: Proceedings of the Symposium on Geometry Processing, pp. 61-70 (2006)

49. Kazhdan, M., Hoppe, H.: Screened poisson surface reconstruction. ACM Trans. Graph. 32(3), 29:1-29:13 (2013). https://doi. org/10.1145/2487228.2487237

50. Kelch, I.D., Bogle, G., Sands, G.B., Phillips, A.R.J., LeGrice, I.J., Rod Dunbar, P.: Organ-wide 3D-imaging and topological analysis of the continuous microvascular network in a murine lymph node. Sci. Rep. 5(1), 16534 (2015). https://doi.org/10.1038/srep16534

51. Keller, L.G.A., Mondino, A., Rivière, T.: Embedded surfaces of arbitrary genus minimizing the willmore energy under isoperimet- ric constraint. Arch. Ration. Mech. Anal. 212(2), 645-682 (2014). https://doi.org/10.1007/s00205-013-0694-9

52. Kelley, C.T.: Iterative methods for optimization. Soc. Ind. Appl. Math. (1999). https://doi.org/10.1137/1.9781611970920

53. Kim, J., Lee, C.O.: Three-dimensional volume reconstruction using two-dimensional parallel slices. SIAM J. Imag. Sci. 12, $1-27$ (2019)

54. Kim, J., Lee, S., Choi, Y., Lee, S.M., Jeong, D.: Basic principles and practical applications of the cahn-hilliard equation. Math. Probl. Eng. 2016, 1-11 (2016). https://doi.org/10.1155/ 2016/9532608

55. Kingma, D.P., Ba, J.: Adam: a method for stochastic optimization. In: International Conference on Learning Representations (2015). arXiv: 1412.6980

56. Kraitzman, N., Promislow, K.: An overview of network bifurcations in the functionalized Cahn-Hilliard free energy. In: J. Bourguignon, R. Jeltsch, A. Pinto, M. Viana (eds.) Mathematics of Energy and Climate Change, CIM Series in Mathematical Sciences, vol. 2. Springer, New York (2015). https://books.google. co.uk/books?id=bNZJCgAAQBAJ

57. Kraitzman, N., Promislow, K.: Pearling bifurcations in the strong functionalized Cahn-Hilliard free energy. arXiv:1711.00396 [math] (2017)

58. Krantz, S.G., Parks, H.R.: Distance to Ck hypersurfaces. J. Differ. Equ. 40(1), 116-120 (1981). https://doi.org/10.1016/00220396(81)90013-9

59. Kumar, S., Tan, S., Zheng, L., Kochmann, D.M.: Inverse-designed spinodoid metamaterials. NPJ Comput. Mater. 6(1), 73 (2020). https://doi.org/10.1038/s41524-020-0341-6

60. Kusner, R.: Comparison surfaces for the Willmore problem. Pacific J. Math. 138(2), 317-345 (1989). https://projecteuclid.org: 443/euclid.pjm/1102650153. Publisher: Pacific Journal of Mathematics, A Non-profit Corporation

61. Kuwert, E., Li, Y., Schätzle, R.: The large genus limit of the infimum of the Willmore energy. Am. J. Math. 132(1), 37-51 (2010). https://doi.org/10.1353/ajm.0.0100. http://muse.jhu.edu/ content/crossref/journals/american_journal_of_mathematics/ v132/132.1.kuwert.html

62. Landy, M.S., Graham, N.: Visual Perception of Texture. In: Chalupa, L.M., Werner, J.S. (eds.) The visual neurosciences, p. 1106. MIT Press, Cambridge, Mass (2004)

63. Lewiner, T., Lopes, H., Vieira, A.W., Tavares, G.: Efficient implementation of marching cubes cases with topological guarantees. J. Graph. Tools 8(2), 1-15 (2003). https://doi.org/10.1080/ 10867651.2003.10487582

64. Li, Y., Shin, J., Choi, Y., Kim, J.: Three-dimensional volume reconstruction from slice data using phase-field models. Comput. Vis. Image Understand. 137, 115-124 (2015). https://doi. org/10.1016/j.cviu.2015.02.001. https://linkinghub.elsevier.com/ retrieve/pii/S1077314215000351

65. Liu, H., Kang, Q., Leonardi, C.R., Schmieschek, S., Narváez, A., Jones, B.D., Williams, J.R., Valocchi, A.J., Harting, J.: Multiphase lattice Boltzmann simulations for porous media applications: a review. Comput. Geosci. 20(4), 777-805 (2016). https://doi.org/ 10.1007/s10596-015-9542-3

66. Loshchilov, I., Hutter, F.: Decoupled weight decay regularization. In: International Conference on Learning Representations (2019). https://openreview.net/forum?id=Bkg6RiCqY7

67. Marques, F.C., Neves, A.: Min-Max theory and the Willmore conjecture. Ann. Math. 179(2), 683-782 (2014)

68. Masnou, S., Morel, J.M.: Level lines based disocclusion. In: Proceedings 1998 International Conference on Image Processing. ICIP98 (Cat. No.98CB36269), vol. 3, pp. 259-263. IEEE Computational Society, Chicago, IL, USA (1998). https://doi.org/10. 1109/ICIP.1998.999016 
69. McInnes, L., Healy, J., Astels, S.: hdbscan: Hierarchical density based clustering. The Journal of Open Source Software 2(11) (2017). https://doi.org/10.21105/joss.00205. Publisher: The Open Journal

70. McInnes, L., Healy, J., Melville, J.: UMAP: Uniform manifold approximation and projection for dimension reduction. arXiv:1802.03426 [cs, stat] (2020)

71. Merveille, O., Miraucourt, O., Salmon, S., Passat, N., Talbot, H.: A variational model for thin structure segmentation based on a directional regularization. In: Proceedings of the 2016 IEEE International Conference on Image Processing (ICIP), pp. 4324-4328 (2016). https://doi.org/10.1109/ICIP.2016.7533176. ISSN: 23818549

72. Miranville, A.: The Cahn-Hilliard equation and some of its variants. AIMS Math. 2(3), 479-544 (2017). https://doi.org/10.3934/ Math.2017.2.479

73. Modica, L., Mortola, S.: Un esempio di Gamma-convergenza. Bollettino della Unione Matematica Italiana B 14, 285-299 (1977)

74. Moser, R.: A higher order asymptotic problem related to phase transitions. SIAM J. Math. Anal. 37(3), 712-736 (2005). https:// doi.org/10.1137/040616760

75. Mumford, D.: Elastica and computer vision. In: C.L. Bajaj (ed.) Algebraic Geometry and its Applications: Collections of Papers from Shreeram S. Abhyankar's 60th Birthday Conference, pp. 491-506. Springer, New York, NY (1994). https://doi.org/10. 1007/978-1-4612-2628-4_31

76. Müller, R.: Hierarchical microimaging of bone structure and function. Nat. Rev. Rheumatol. 5(7), 373-381 (2009). https://doi.org/ 10.1038/nrrheum.2009.107. Number: 7 Publisher: Nature Publishing Group

77. Naboni, R., Kunic, A.: Design and additive manufacturing of lattice-based cellular solids at building scale (2017). https://doi. org/10.5151/sigradi2017-058. Pages: 375

78. Nagase, Y., Tonegawa, Y.: A singular perturbation problem with integral curvature bound. Hiroshima Math. J. 37(3), 455489 (2007). https://doi.org/10.32917/hmj/1200529813. Publisher: Hiroshima University, Mathematics Program

79. Niethammer, M., Stein, A.N., Kalies, W.D., Pilarczyk, P., Mischaikow, K., Tannenbaum, A.: Analysis of blood vessel topology by cubical homology. In: Proceedings of the International Conference on Image Processing, vol. 2, pp. II-II. IEEE (2002)

80. Osher, S., Fedkiw, R.: Level Set Methods and Dynamic Implicit Surfaces. Applied Mathematical Sciences. Springer, New York (2002)

81. Parfitt, A.M.: Trabecular bone architecture in the pathogenesis and prevention of fracture. Am. J. Med. 82(1), 68-72 (1987). https:// doi.org/10.1016/0002-9343(87)90274-9. https://www.amjmed. com/article/0002-9343(87)90274-9/abstract. Publisher: Elsevier

82. Passaro, D., Tullio, A.D., Abarrategi, A., Rouault-Pierre, K., Foster, K., Ariza-McNaughton, L., Montaner, B., Chakravarty, P., Bhaw, L., Diana, G., Lassailly, F., Gribben, J.G., Bonnet, D.: Increased vascular permeability in the bone marrow microenvironment contributes to disease progression and drug response in acute Myeloid Leukemia. Cancer cell (2017)

83. Paszke, A., Gross, S., Massa, F., Lerer, A., Bradbury, J., Chanan, G., Killeen, T., Lin, Z., Gimelshein, N., Antiga, L., Desmaison, A., Kopf, A., Yang, E., DeVito, Z., Raison, M., Tejani, A., Chilamkurthy, S., Steiner, B., Fang, L., Bai, J., Chintala, S.: PyTorch: an imperative style, high-performance deep learning library. In: H. Wallach, H. Larochelle, A. Beygelzimer, F. d'Alché Buc, E. Fox, R. Garnett (eds.) Advances in neural information processing systems 32, pp. 8024-8035. Curran Associates, Inc. (2019)

84. Piccinelli, M., Veneziani, A., Steinman, D.A., Remuzzi, A., Antiga, L.: A framework for geometric analysis of vascular structures: application to cerebral aneurysms. IEEE Trans. Med. Imag. 28, 1141-1155 (2009)
85. Portilla, J., Simoncelli, E.P.: A parametric texture model based on joint statistics of complex wavelet coefficients. Int. J. Comput. Vis. 40(1), 49-71 (2000)

86. Pozrikidis, C.: Numerical simulation of blood flow through microvascular capillary networks. Bull. Math. Biol. 71(6), 1520 1541 (2009). https://doi.org/10.1007/s11538-009-9412-z

87. Puhka, M., Joensuu, M., Vihinen, H., Belevich, I., Jokitalo, E.: Progressive sheet-to-tubule transformation is a general mechanism for endoplasmic reticulum partitioning in dividing mammalian cells. Mol. Biol. Cell 23(13), 2424-2432 (2012). https:// doi.org/10.1091/mbc.e10-12-0950

88. Ramasamy, S.K.: Structure and functions of blood vessels and vascular niches in bone. Stem Cells Int. (2017). https://doi.org/ $10.1155 / 2017 / 5046953$

89. Rust, R., Kirabali, T., Grönnert, L., Dogancay, B., Limasale, Y.D.P., Meinhardt, A., Werner, C., Laviña, B., Kulic, L., Nitsch, R.M., Tackenberg, C., Schwab, M.E.: A practical guide to the automated analysis of vascular growth, maturation and injury in the brain. Front. Neurosci. 14, 244 (2020). https://doi.org/10. 3389/fnins.2020.00244

90. Ryan, T.M., Shaw, C.N.: Unique suites of trabecular bone features characterize locomotor behavior in human and non-human anthropoid primates. PLoS ONE 7(7), e41037 (2012). https://doi. org/10.1371/journal.pone.0041037

91. Röger, M., Schätzle, R.: On a modified conjecture of De Giorgi. Math. Z. 254(4), 675-714 (2006). https://doi.org/10.1007/ s00209-006-0002-6

92. Salmon, P.: Non-linear pattern formation in bone growth and architecture. Front. Endocrinol. 5 (2015). https://doi.org/10.3389/ fendo.2014.00239. Publisher: Frontiers

93. Salmon, P.L., Ohlsson, C., Shefelbine, S.J., Doube, M.: Structure model index does not measure rods and plates in trabecular bone. Front. Endocrinol. 6,(2015). https://doi.org/10.3389/fendo.2015. 00162. Publisher: Frontiers

94. Sarker, M., Naghieh, S., Sharma, N., Chen, X.: 3D biofabrication of vascular networks for tissue regeneration: a report on recent advances. J. Pharm. Anal. 8(5), 277-296 (2018). https://doi. org/10.1016/j.jpha.2018.08.005. https://linkinghub.elsevier.com/ retrieve/pii/S2095177918300911

95. Scheidweiler, D., Miele, F., Peter, H., Battin, T.J., de Anna, P.: Trait-specific dispersal of bacteria in heterogeneous porous environments: from pore to porous medium scale. J. R. Soc. Interface 17(164), 20200046 (2020). https://doi.org/10.1098/rsif. 2020.0046

96. Schwarz, D.S., Blower, M.D.: The endoplasmic reticulum: structure, function and response to cellular signaling. Cell. Mol. Life Sci. 73(1), 79-94 (2016). https://doi.org/10.1007/s00018-0152052-6

97. Seguin, B., Fried, E.: Microphysical derivation of the CanhamHelfrich free-energy density. J. Math. Biol. 68(3), 647-665 (2014). https://doi.org/10.1007/s00285-013-0647-9

98. Seifert, U.: Configurations of fluid membranes and vesicles. Adv. Phys. 46(1), 13-137 (1997). https://doi.org/10.1080/ 00018739700101488. Publisher: Taylor \& Francis _eprint

99. Shen, J., Kang, S.H., Chan, T.F.: Eulers elastica and curvaturebased inpainting. SIAM J. Appl. Math. 63(2), 564-592 (2003). https://doi.org/10.1137/S0036139901390088

100. Simon, L.: Existence of surfaces minimizing the Willmore functional. Commun. Anal. Geometry 1(2), 281-326 (1993). https:// doi.org/10.4310/CAG.1993.v1.n2.a4. https://www.intlpress. com/site/pub/pages/journals/items/cag/content/vols/0001/0002/ a004/index.php. Publisher: International Press of Boston

101. Sivaraj, K.K., Adams, R.H.: Blood vessel formation and function in bone. Development 143(15), 2706-15 (2016) 
102. Séjourné, T., Feydy, J., Vialard, F.X., Trouvé, A., Peyré, G.: Sinkhorn divergences for unbalanced optimal transport. arXiv:1910.12958 [cs, math, stat] (2019)

103. Tamada, T., Sone, T., Jo, Y., Imai, S., Kajihara, Y., Fukunaga, M.: Three-dimensional trabecular bone architecture of the lumbar spine in bone metastasis from prostate cancer: comparison with degenerative sclerosis. Skeletal Radiol. 34(3), 149-155 (2005). https://doi.org/10.1007/s00256-004-0855-x

104. Toda, M.: The Willmore conjecture and the Willmore energy. Chapman \& Hall/CRC monographs and research notes in mathematics. CRC Press, Boca Raton (2018)

105. Tonegawa, Y.: Phase field model with a variable chemical potential. Proc. R. Soc. Edinb. Sect. A Math. 132(4), 993-1019 (2002). https://doi.org/10.1017/S0308210500001980. Publisher: Royal Society of Edinburgh Scotland Foundation

106. Tu, Z.C., Ou-Yang, Z.C.: A geometric theory on the elasticity of bio-membranes. J. Phys. A Math. Gen. 37(47), 11407-11429 (2004). https://doi.org/10.1088/0305-4470/37/47/ 010. Publisher: IOP Publishing

107. Tyrrell, J.A., Tomaso, E.d., Fuja, D., Tong, R., Kozak, K., Jain, R.K., Roysam, B.: Robust 3-D modeling of vasculature imagery using superellipsoids. IEEE Trans. Med. Imag. 26(2), 223-237 (2007). https://doi.org/10.1109/TMI.2006.889722. Conference Name: IEEE Transactions on Medical Imaging
108. Vacher, J., Briand, T.: The Portilla-Simoncelli texture model: towards the understanding of the early visual cortex (2020)

109. Von Der Schulenburg, D.G., Pintelon, T., Picioreanu, C., Van Loosdrecht, M., Johns, M.: Three-dimensional simulations of biofilm growth in porous media. AIChE J. 55(2), 494-504 (2009). Publisher: Wiley Online Library

110. Willmore, T.J.: Riemannian geometry. Oxford University Press, Oxford (1996)

111. Xia, L., Breitkopf, P.: Recent advances on topology optimization of multiscale nonlinear structures. Arch. Comput. Methods Eng. 24(2), 227-249 (2017). https://doi.org/10.1007/s11831016-9170-7

Publisher's Note Springer Nature remains neutral with regard to jurisdictional claims in published maps and institutional affiliations. 